Systematic Review

\title{
Mapping the Association between Vitamin D and Low Back Pain: A Systematic Review and Meta- Analysis of Observational Studies
}

\footnotetext{
Joshua Zadro, BAppSc1, Debra Shirley, PhD¹, Manuela Ferreira, PhD², Ana Paula Carvalho-Silva, MSc ${ }^{1}$, Sarah E. Lamb, PhD³, Cyrus Cooper, PhD, and Paulo H. Ferreira, PhD ${ }^{1}$

From: ${ }^{2}$ Discipline of Physiotherapy, Faculty of Health Sciences, The University of Sydney, Sydney, Australia; ${ }^{2}$ Institute of Bone and Joint Research/ The Kolling Institute \& School of Public Health, Sydney Medical School, The University of Sydney, Sydney, Australia;

Nuffield Department of Orthopaedics, Rheumatology and Musculoskeletal Sciences, University of Oxford, Oxford, England

Address Correspondence: Joshua Zadro, BAppSc The University of Sydney, Discipline of Physiotherapy 75 East St, Lidcombe Sydney NSW 1825, Australia E-mail:

jzad3326@uni.sydney. edu.au

Disclaimer: There was no external funding in the preparation of this manuscript.

Conflict of interest: Each author certifies that he or she, or a member of his or her immediate family, has no commercial association

(i.e., consultancies, stock ownership, equity interest, patent/licensing arrangements, etc.) that might pose a conflict of interest in connection with the submitted manuscript.

Manuscript received: 03-28-2017

Revised manuscript received: 05-22-2017

Accepted for publication: 05-30-2017

Free full manuscript: www.painphysicianjournal.com

Background: Low back pain (LBP) is the highest contributor to disability worldwide, with current intervention strategies only providing small to moderate analgesic effects. The use of vitamin $D$ supplementation for LBP has gained interest due to its proposed anti-inflammatory and neuromodulatory properties. However, it is still unclear whether vitamin D levels differ between those with and without LBP or if vitamin $\mathrm{D}$ levels are associated with pain intensity.

Objectives: We aim to investigate the association between vitamin D levels and LBP and to determine if vitamin D levels correlate with pain intensity in individuals with LBP.

Study Design: This study was conducted in accordance with the guidelines for performing a Metaanalysis and Systematic Review Of Observational Studies in Epidemiology (MOOSE).

Methods: We performed electronic database searches combined keywords relating to vitamin $D$ and LBP in MEDLINE, CINAHL, EMBASE, AMED, WEB OF SCIENCE, and SCOPUS from the earliest record to March 2017. Studies were included if they reported any quantitative measure of vitamin $D$, such as serum 25-hydroxyvitamin D [25(OH)D], with adequate data in patients with and without LBP or adequate data on pain intensity in patients with LBP. No restriction on the type or duration of LBP, nor the age and gender of patients was applied. Two reviewers independently performed the selection of studies, extracted data, and assessed the methodological quality of the included studies using a modified 15-item Downs and Black checklist.

Results: After the removal of duplicates and the screening of titles and abstracts, 105 full texts were evaluated. There were 29 articles included in this systemic review (22 entered into a meta-analysis), including 19 cross-sectional studies, 9 case-control studies, and one single-arm surgical trial where the pre-operative data were used in our analyses. The pooled results from 19 studies showed that individuals with LBP were more likely to have vitamin D deficiency (pooled $\mathrm{OR}=1.60,95 \% \mathrm{Cl}: 1.20-2.12, P=0.001$, $n=19$ ), severe deficiency (pooled $O R=2.08,95 \% C l: 1.19-3.64, P=0.010, n=7$ ), and lower serum concentrations of $25(\mathrm{OH}) \mathrm{D}$ (weighted $\mathrm{MD}=3.86,95 \% \mathrm{Cl}: 0.20-7.52, P=0.039, \mathrm{n}=12$ ) compared to those without LBP (where " $\mathrm{n}$ " is the number of studies). The association between vitamin $D$ deficiency (pooled $\mathrm{OR}=1.83,95 \% \mathrm{Cl}: 1.26-2.66, P=0.002, \mathrm{n}=9$ ) or serum 25(OH)D (weighted $\mathrm{MD}=7.64$, $95 \% \mathrm{Cl}: 4.02-11.26, P<0.001, \mathrm{n}=4$ ) and LBP was stronger for women but failed to be statistically significant for men (pooled $\mathrm{OR}=1.06,95 \% \mathrm{Cl}: 0.62-1.81, P=0.213, \mathrm{n}=3$ ). In addition, there were strong associations between vitamin D deficiency and LBP in patients $<60$ years old (particularly women). We found minimal evidence to support an association between vitamin D levels and pain intensity in patients with LBP.

Limitations: We were unable to investigate whether vitamin D deficiency increases the risk of developing LBP as there were no longitudinal studies included in this review.

Conclusion: Vitamin D deficiency is associated with LBP, with stronger associations observed in younger women and those with severe levels of deficiency. The association between vitamin $D$ levels and pain intensity is inconsistent. These results may guide the implementation of future studies on vitamin $D$ supplementation for LBP.

PROSPERO Registration No: CRD42016046874

Key words: Vitamin D, low back pain, deficiency, pain intensity, serum 25-hydroxyvitamin D, supplementation, cross-sectional study, case-control study

Pain Physician 2017; 20:611-640
} 
L

ow back pain (LBP) is a global problem, being the highest contributor of years lived with disability in both developed and developing countries (1). From an economic perspective, the burden of LBP can be seen across many countries $(2,3)$, with the annual cost of LBP in Australia estimated around $\$ 5$ billion (4). The majority of research on LBP has concerned management strategies; however, given the small effect sizes of current interventions (5), a better understanding of the factors associated with the prevalence and risk of developing LBP is needed to guide future intervention strategies.

A previous history of LBP appears to be the only strong and consistent risk factor for developing LBP (6), with other factors only demonstrating weak associations, including obesity (7), heavy work-related physical activity (8), poor general health (9), educational attainment (10), and symptoms of depression (11). A number of studies have demonstrated an association between vitamin $D$ deficiency and the presence of chronic painful conditions (12-15). However, the evidence surrounding vitamin $D$ levels in people with and without LBP, along with how vitamin $D$ levels influence pain intensity in patients with LBP appears to be conflicting. Some studies show a positive association between vitamin $D$ deficiency and $\operatorname{LBP}(16,17)$ and between vitamin $D$ levels and pain intensity $(18,19)$, while others have failed to find an association (20) or have only found a significant association in women (21).

There are numerous mechanisms that provide rationale for the link between vitamin $D$ and the risk of LBP, including: the regulation of anti- and pro-inflammatory cytokines that control pain and inflammation (22) and the modulation of pain through sensory neuron excitability $(23,24)$. Furthermore, there appears to be an inverse relationship between inflammatory markers and serum concentrations of 25-hydroxyvitamin D (25(OH)D) (a common measure of vitamin D levels) (25), with research showing reductions in inflammatory markers following vitamin D supplementation $(26,27)$. Therefore, given the increasing interest in vitamin $D$ supplementation for the management of $\operatorname{LBP}(28,29)$, a better understanding of the relationship between vitamin $D$ levels and LBP is needed. The aim of this systematic review is to investigate if vitamin $D$ levels are associated with the prevalence and risk of LBP and if vitamin D levels correlate with pain intensity in patients with LBP.

\section{MethodS}

\section{Search Strategy}

The protocol for this systematic review was registered on PROSPERO (Registration No: CRD42016046874) and was conducted in accordance with the Preferred Reporting Items for Systematic Reviews and MetaAnalyses (PRISMA) statement (30) and the guidelines for performing a Meta-analysis and Systematic Review Of Observational Studies in Epidemiology (MOOSE) (31). We performed electronic database searches (from the earliest records to March 2017) combing key words relating to vitamin D (e.g., "alfacalcidol" OR "ergocalciferol" OR "1-alpha hydroxyvitamin D3," etc.) and LBP (e.g., "back ache" OR "back pain" OR "spinal pain," etc.) in MEDLINE, CINAHL, EMBASE, AMED, WEB OF SCIENCE and SCOPUS databases (Appendix 1). We performed citation tracking and hand-searched the reference lists of included studies to capture articles missed through our database search.

\section{Study Selection}

Two reviewers (JZ and AS) independently performed the selection of studies by screening the titles, abstracts, and full texts of articles. Both of the reviewers used a study eligibility form based on items from the inclusion/exclusion criteria and resolved any disagreements by discussion or consultation with a third reviewer (DS). Studies in a language other than English needed to be translated or at least have the abstract in English to be included. We included conference abstracts or the abstracts of articles where the full text was not available.

Studies reporting the association between vitamin D levels [serum concentrations of $25(\mathrm{OH}) \mathrm{D}$ or the presence of vitamin D deficiency] and LBP (or pain intensity in people with LBP) or with adequate data to calculate a relevant measure of association [mean difference (MD), odds ratio (OR), Pearson's correlation coefficient $(r)$, or Spearman's rho $(\nabla)]$ were included. There was no restriction on the duration or type of LBP assessed (e.g., non-specific LBP or LBP with radicular symptoms), the cut-off for vitamin $D$ deficiency used, the measure of pain intensity, nor the age or gender of the patients. We included longitudinal and cross-sectional studies, case-control studies, and caseseries that fit the above inclusion criteria. We excluded studies investigating the effectiveness of vitamin $D$ supplementation for LBP, which includes randomized and non-randomized trials. 


\section{Methodological Quality}

The methodological quality of the included studies with an accessible full text was independently assessed by 2 reviewers (JZ and AS) using a modified Downs and Black checklist (32). Any disagreement was resolved by discussion or consultation with a third reviewer (DS).

\section{Data Extraction}

Two reviewers (JZ and AS) used a standardized data extraction form to independently extract the data from the included studies. The data on the patients' characteristics (age and gender), study geographical location, study setting (e.g., hospital or community), sample size, and features specific to the study design, such as the exposure variables [e.g., serum 25(OH)D], outcome variables (e.g., LBP), pain intensity measures, and confounders, were extracted.

\section{Statistical Analysis}

The data on the most relevant statistics to measure the association between vitamin D and LBP (mean difference [MD] or odds ration [OR]) or association between vitamin $\mathrm{D}$ and pain intensity in patients with LBP (MD, OR, $r, \mathbb{Q})$ and their $95 \%$ confidence interval $(\mathrm{Cl})$ were extracted from the included studies. When studies were considered sufficiently homogenous we attempted to pool the results using the most adjusted models reported in the studies using Comprehensive Meta-Analysis Version 3.0 (BioStat, Englewood, NJ). If more than half of the studies failed to adjust their results for potential confounders, we stratified our meta-analyses by age, gender, and cut-offs for vitamin $\mathrm{D}$ deficiency where possible (sensitivity analysis). We attempted to transform data into MD and their $95 \%$ $\mathrm{Cl}$ when studies only reported: i) the mean (standard deviation [SD]) serum 25(OH)D in people with and without LBP, ii) the mean (SD) serum 25(OH)D in people with LBP who had varying levels of pain intensity, or iii) the mean (SD) pain intensity in people with and without vitamin D deficiency. In addition, we attempted to transform data into OR and their $95 \% \mathrm{Cl}$ when studies only reported: i) the number of patients with and without LBP who were vitamin $\mathrm{D}$ deficient (or severely deficient) or ii) the number of individuals with severe or mild pain who were vitamin D deficient. The authors of the included studies were contacted when required data was not published. We assessed heterogeneity using the $1^{2}$ statistic and considered $\mathrm{I}^{2}<25 \%, \mathrm{I}^{2} \geq 50 \%$, and $\mathrm{I}^{2} \geq 75 \%$ as indications of low, moderate, and high heterogeneity respectively (33). When $\mathrm{I}^{2}$ was < $50 \%$ we used fixed-effects models, and if it was $\geq 50 \%$ we used random-effects models.

\section{Results}

\section{Description of Studies}

We identified 3,534 articles through our database searches with 3 articles (34-36) identified through hand-searching the reference lists of the included studies (Fig. 1). Following the removal of duplicates, 2 reviewers ( $J Z$ and $A S$ ) independently screened the articles' titles and abstracts and screened the full-text of 105 articles. A total of 29 observational studies (with data on 21,764 patients) were eligible for inclusion in this review, including 19 cross-sectional studies (including 4 conference abstracts), 9 case-control studies, and one single-arm surgical trial where we used the pre-operative data in our analyses. The full texts of 2 included case-control studies $(37,38)$ could not be obtained, thus we used the data included in the abstracts for our review. There were no longitudinal studies that met our inclusion criteria. The characteristics of the included studies can be found in Table 1. The duration of LBP symptoms included in the studies varied, with 17 of the 29 studies including patients who reported LBP of any duration (8 studies only included patients with non-specific LBP) and 8 studies including patients with chronic LBP (4 studies only included patients with nonspecific chronic LBP). We defined chronic LBP as pain lasting for at least 3 months (39) and operationalized this over all of the studies, regardless of the authors' classification. The remaining 4 studies included patients with lumbar spinal stenosis $(n=1)$, patients seeking spinal surgery $(n=2)$, or patients reporting ongoing symptoms following spinal surgery $(n=1)$. The majority of the included studies were conducted in the MiddleEast/Mediterranean region $(n=12)$, with the remaining studies being conducted in Europe $(n=3)$, India ( $n=$ $3)$, United Kingdom ( $n=3)$, Korea $(n=2)$, Thailand ( $n$ $=1)$, Japan $(n=1)$, Brazil $(n=1)$, United States ( $n=$ $1)$, Australia $(n=1)$, or across numerous countries ( $n$ = 1) (16) (Table 1). There were differences between the setting for each study with the majority of studies recruiting patients from either health/medical centers or outpatient/rheumatology clinics $(n=16)$ (Table 1). The sample size of individual studies ranged from 9 to 9,305 patients. In cross-sectional studies investigating vitamin $D$ levels in people with and without LBP, the prevalence of LBP ranged from $3.5 \%$ to $74 \%$. The classification of serum 25(OH)D was similar across studies, with 19 studies using serum $25(\mathrm{OH}) \mathrm{D}<20 \mathrm{ng} / \mathrm{mL}$ as an indicator of deficiency, 9 studies using serum $25(\mathrm{OH})$ D between $20-30 \mathrm{ng} / \mathrm{mL}$ as an indicator of insufficiency, and 9 studies considering serum 25(OH)D > 30 


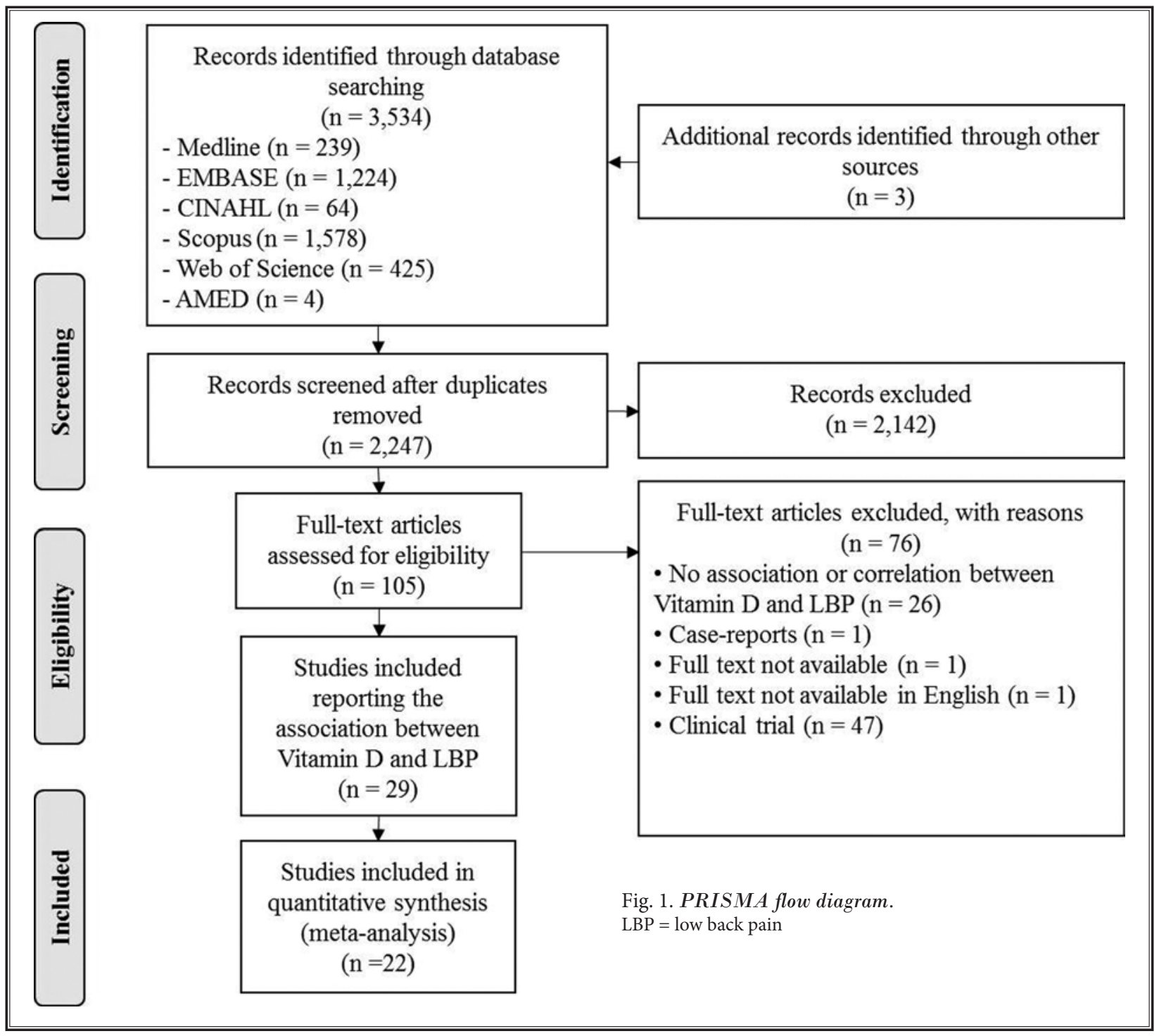

$\mathrm{ng} / \mathrm{mL}$ as normal. Despite the remaining studies using different cut-off values (Table 1), we decided to base the terminology in our review on the above values, as these cut-offs are most commonly used in the vitamin D literature $(40,41)$. Furthermore, the most common cut-off for severe deficiency was $<10 \mathrm{ng} / \mathrm{mL}$ (4 studies) (range: $5-15 \mathrm{ng} / \mathrm{mL}$ ), so we decided to use this value throughout the manuscript.

\section{Methodological Quality}

The original Downs and Black checklist contained 27 items (32). However, since our review only included cross-sectional observational studies, we removed checklist items only relevant to longitudinal studies or clinical trials. The remaining 15 items provided an overall score for study quality based on 5 categories: study quality ( 7 items), external validity ( 2 items), study bias (3 items), and confounding and selection bias (3 items) (Appendix 2). Individual study scores ranged from 8 - 14 (out of a possible 16) with a mean score of 10.7 (Appendix 2). The main methodological limitations included: failing to describe $(n=14)$ and adjust for potential confounders ( $n=20$ ), failing to report that patients willing to participate were representative of the entire population from which they were recruited ( $n=22)$, and not specifying whether any of the results presented were based on "data-dredging" $(n=23)$. 


\begin{tabular}{|c|c|c|c|c|c|c|c|c|c|c|}
\hline 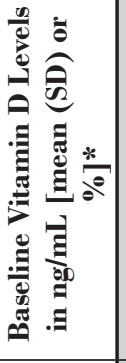 & 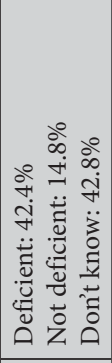 & 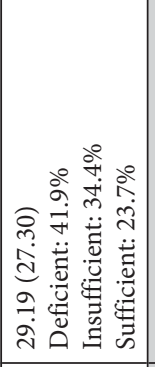 & 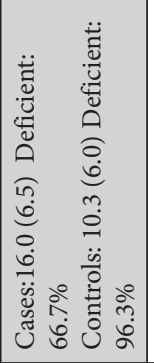 & 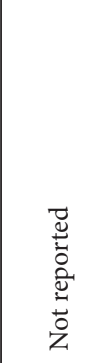 & 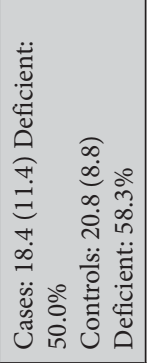 & 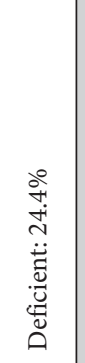 & $\begin{array}{l}\underset{\overbrace{}}{\Xi} \\
\underset{\exists}{ \pm} \\
\stackrel{\infty}{\rightarrow}\end{array}$ & 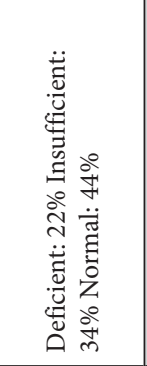 & 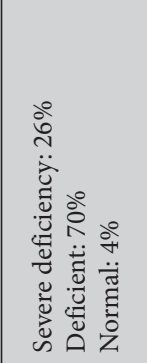 & 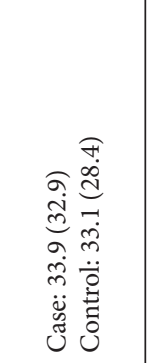 \\
\hline 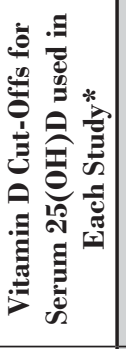 & 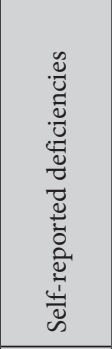 & 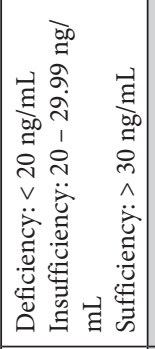 & 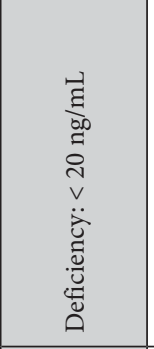 & 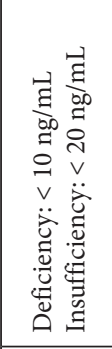 & 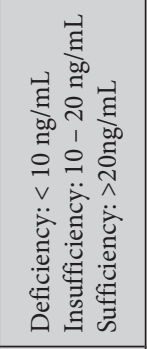 & 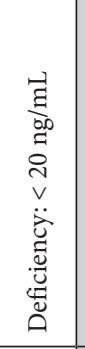 & 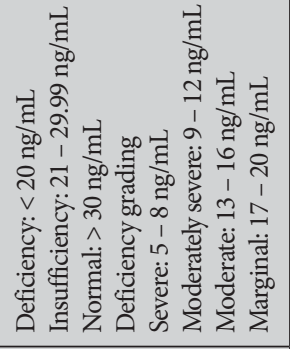 & 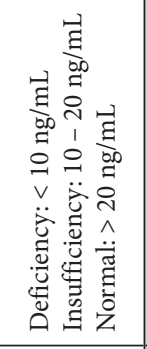 & 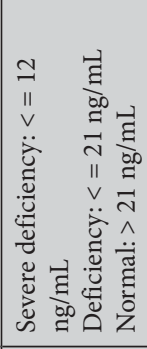 & 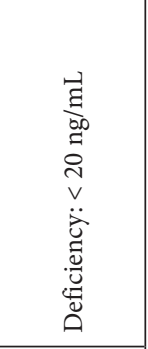 \\
\hline 苞 & 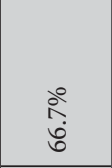 & 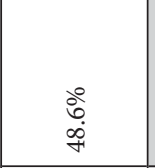 & 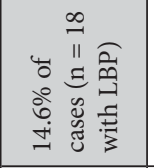 & 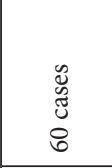 & ì & $\begin{array}{l}\text { ì } \\
\text { in } \\
\text { D }\end{array}$ & 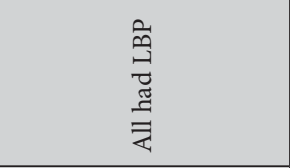 & 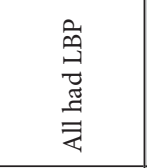 & هì & 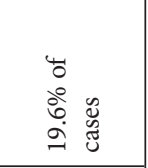 \\
\hline 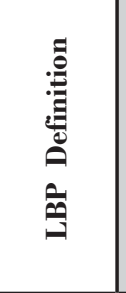 & 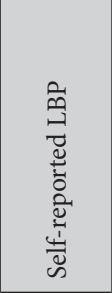 & 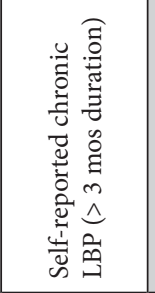 & 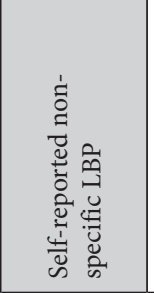 & 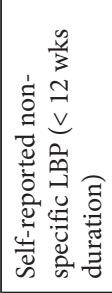 & 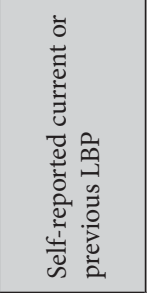 & 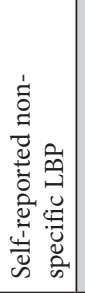 & 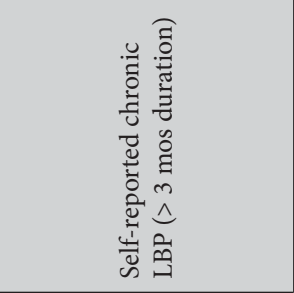 & 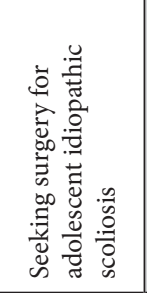 & 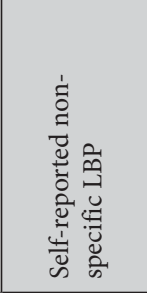 & 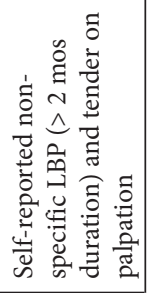 \\
\hline$=$ & $\begin{array}{l}\infty \\
\stackrel{\alpha}{+}\end{array}$ & 命 & ఃั & 8 & $\stackrel{2}{2}$ & 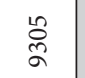 & ల్ & $F$ & $\overrightarrow{\widetilde{N}}$ & 总 \\
\hline 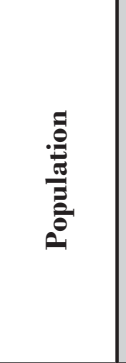 & 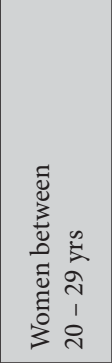 & 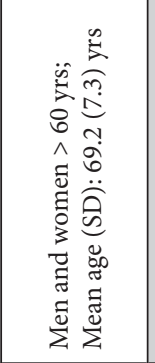 & 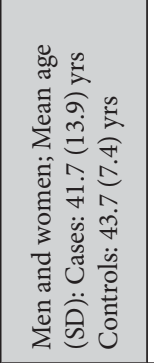 & 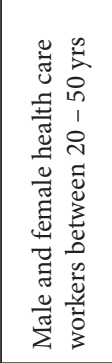 & 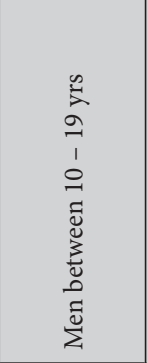 & 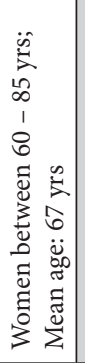 & 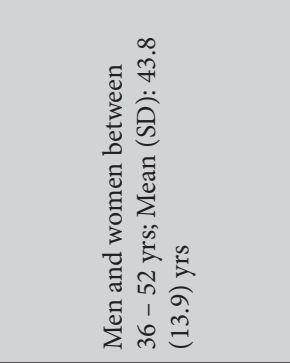 & 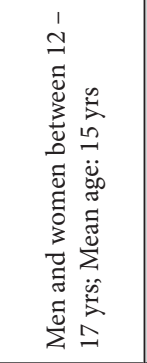 & 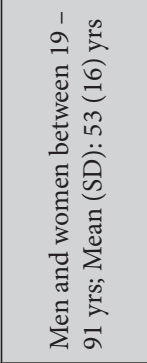 & 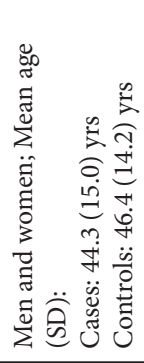 \\
\hline 易 & 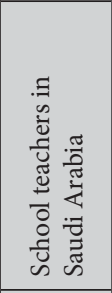 & 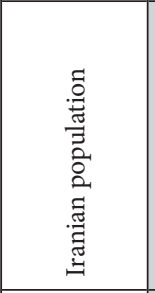 & 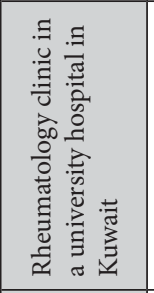 & 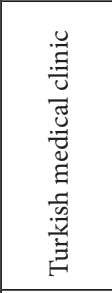 & 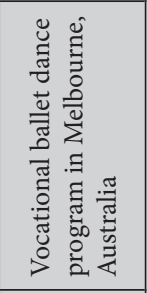 & 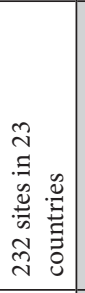 & 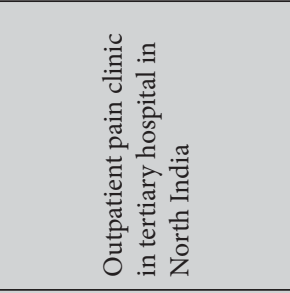 & 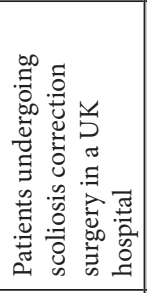 & 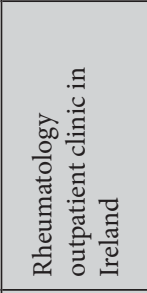 & 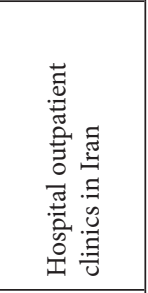 \\
\hline $\begin{array}{l}\widehat{E} \\
\hat{\bar{E}} \\
\hat{\Xi}\end{array}$ & 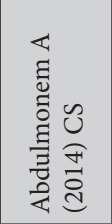 & 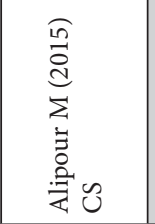 & 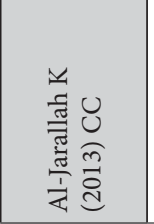 & 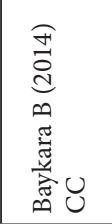 & 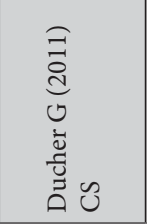 & 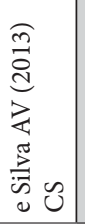 & 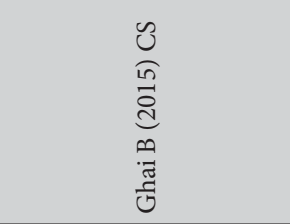 & 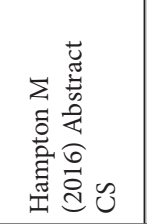 & 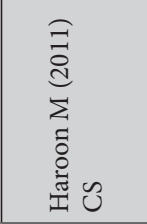 & 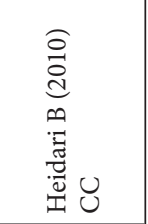 \\
\hline
\end{tabular}




\begin{tabular}{|c|c|c|c|c|c|c|c|c|c|c|}
\hline 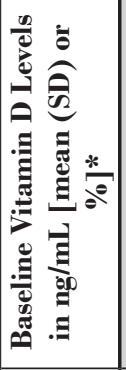 & 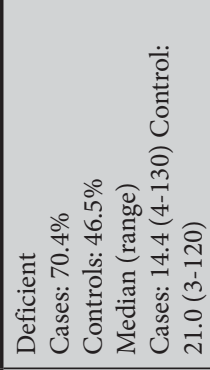 & 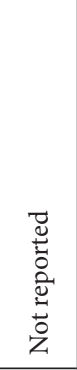 & 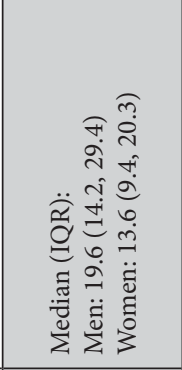 & 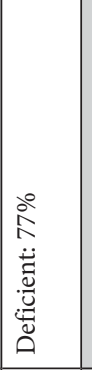 & 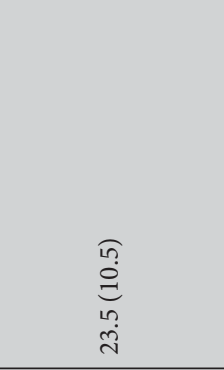 & 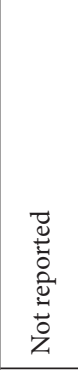 & 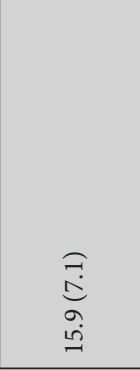 & $\stackrel{\infty}{\text { ஸे }}$ & 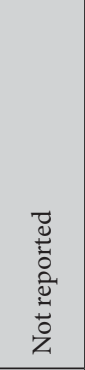 & 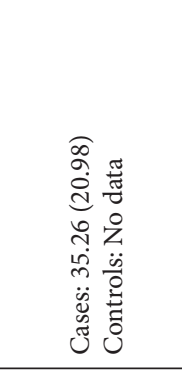 \\
\hline 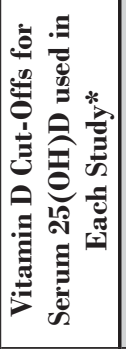 & 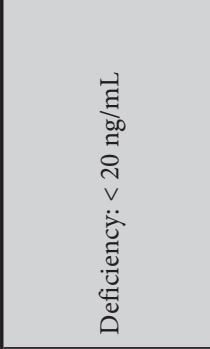 & 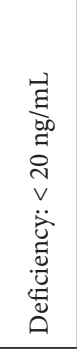 & 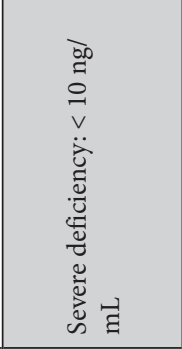 & 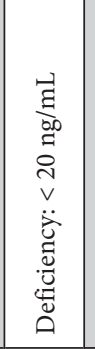 & 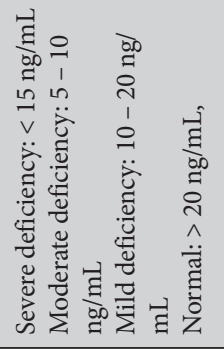 & 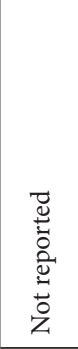 & 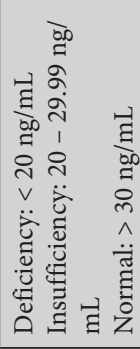 & 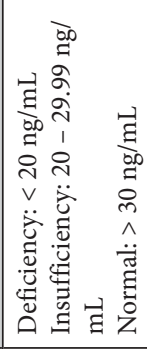 & 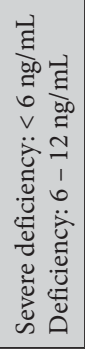 & 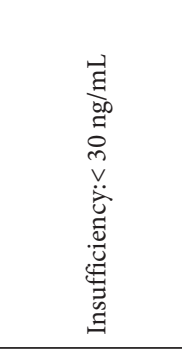 \\
\hline 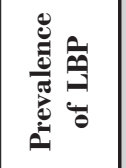 & $\begin{array}{l}\mathscr{J} \\
\tilde{J} \\
\tilde{~} \\
\infty\end{array}$ & $\stackrel{\infty}{\stackrel{\infty}{+}}$ & 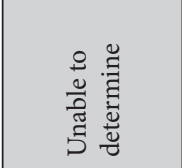 & $\begin{array}{l}\stackrel{\circ}{+}+{ }_{\vec{b}}^{\circ}\end{array}$ & 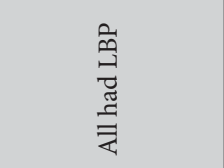 & 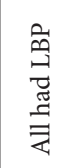 & 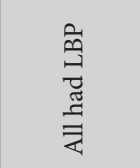 & 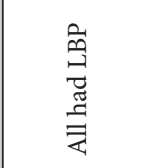 & 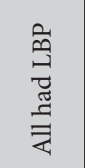 & 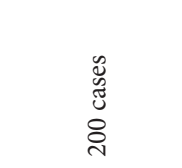 \\
\hline 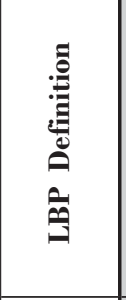 & 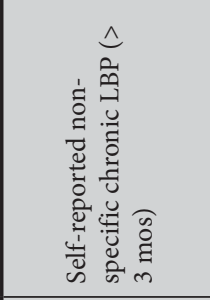 & 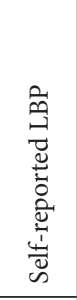 & 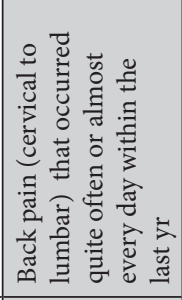 & 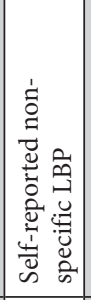 & 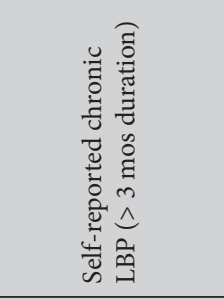 & 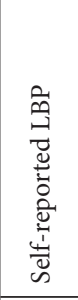 & 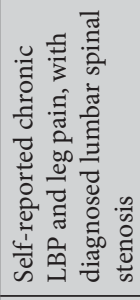 & 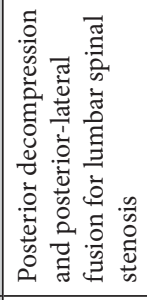 & 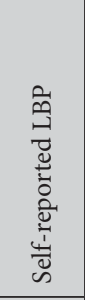 & 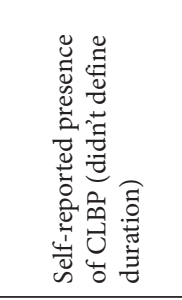 \\
\hline$\Rightarrow$ & $\stackrel{\infty}{\rightarrow}$ & $\stackrel{\Re}{\stackrel{\leftrightarrow}{f}}$ & ڤn & สิ & $\cong$ & సิ & 足 & $\vec{m}$ & $\begin{array}{l}\vec{b} \\
\text { m. }\end{array}$ & \& \\
\hline & 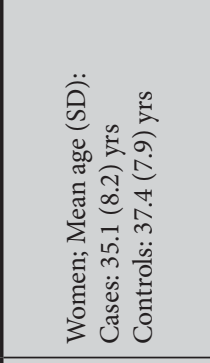 & 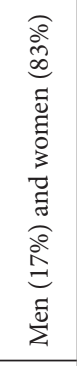 & 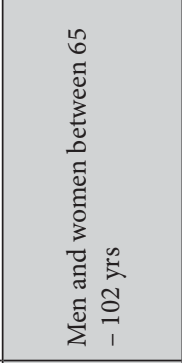 & 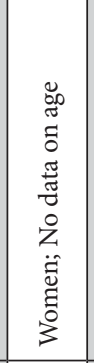 & 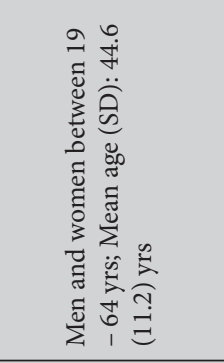 & 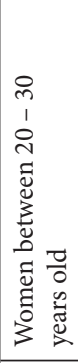 & 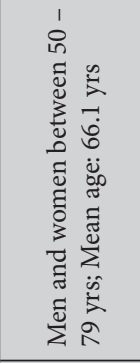 & 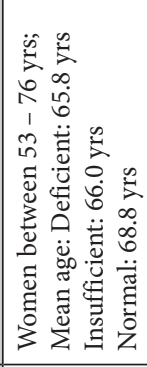 & 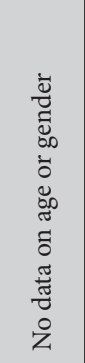 & 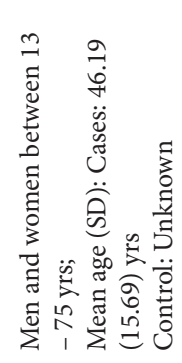 \\
\hline 贺 & 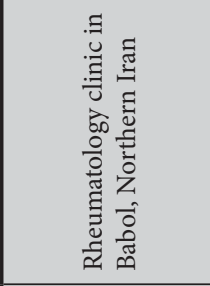 & 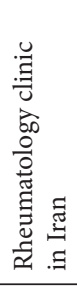 & 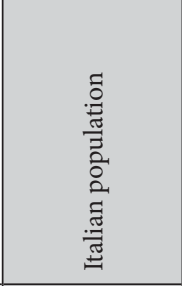 & 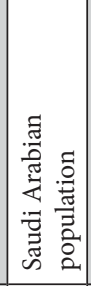 & 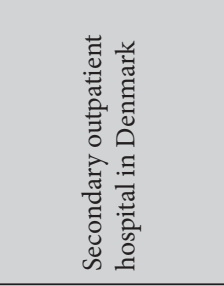 & 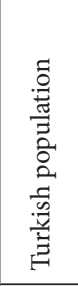 & 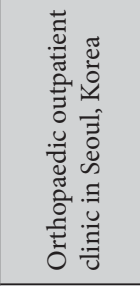 & 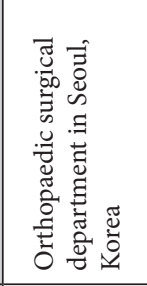 & 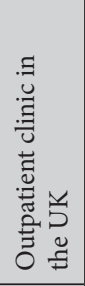 & 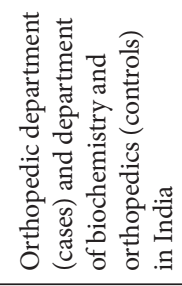 \\
\hline $\begin{array}{l}\widehat{E} \\
\hat{\bar{E}} \\
\hat{\bar{E}}\end{array}$ & 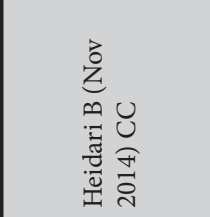 & 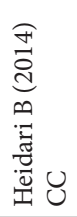 & 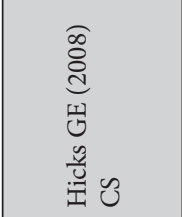 & 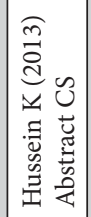 & 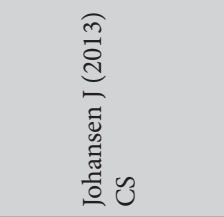 & 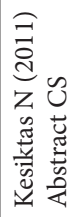 & 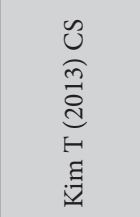 & 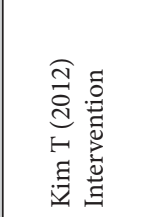 & 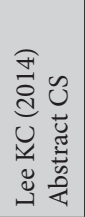 & 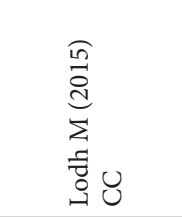 \\
\hline
\end{tabular}


Vitamin D and Low Back Pain

\begin{tabular}{|c|c|c|c|c|c|c|c|c|c|}
\hline 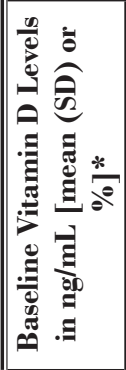 & 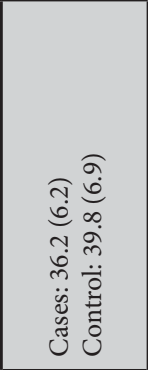 & 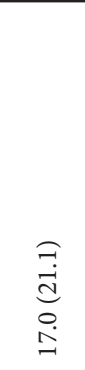 & 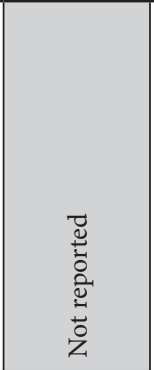 & 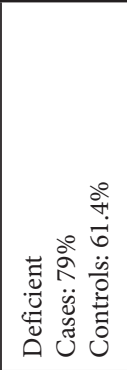 & 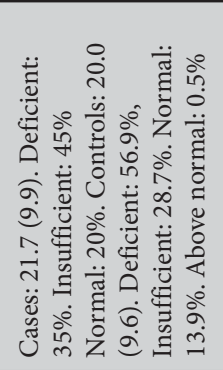 & 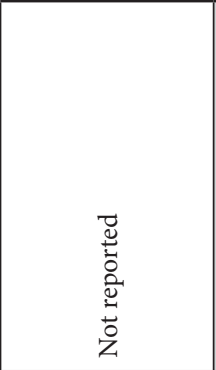 & 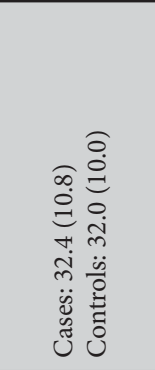 & 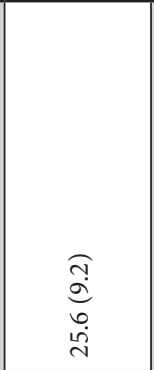 & 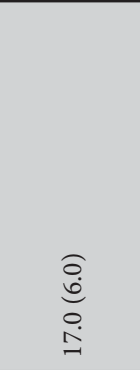 \\
\hline 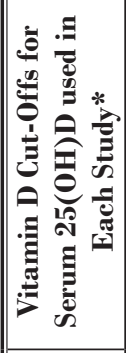 & 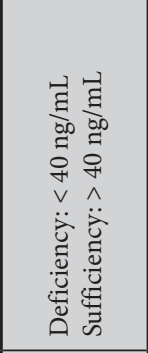 & 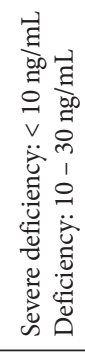 & 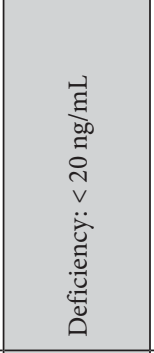 & 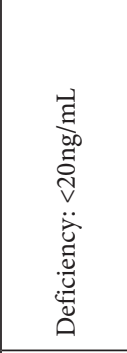 & 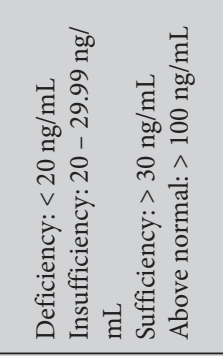 & 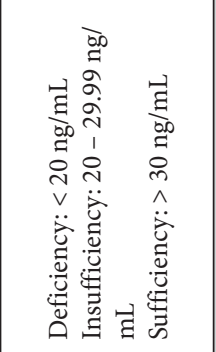 & 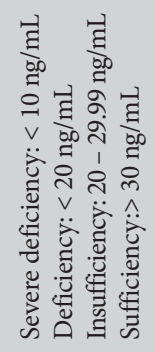 & 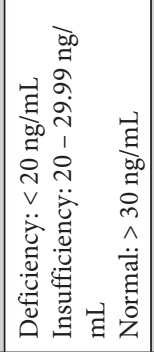 & 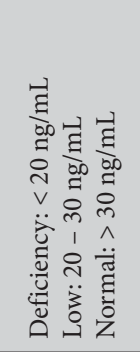 \\
\hline 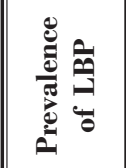 & $\begin{array}{l}\mathscr{8} \\
\tilde{~} \\
\tilde{U} \\
8\end{array}$ & ஓे & ஓे & 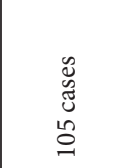 & $\frac{20}{6}$ & 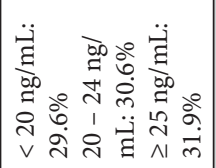 & 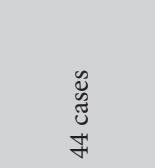 & 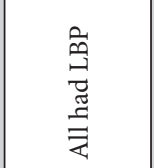 & 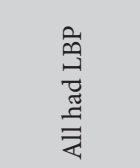 \\
\hline 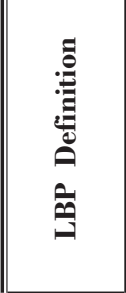 & 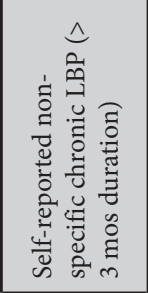 & 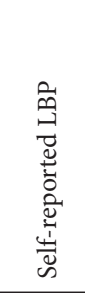 & 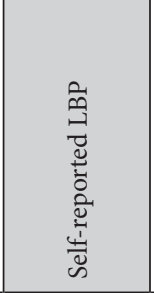 & 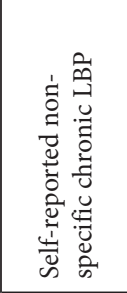 & 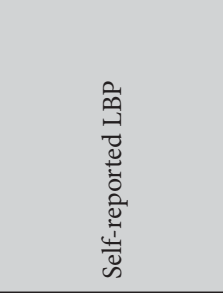 & 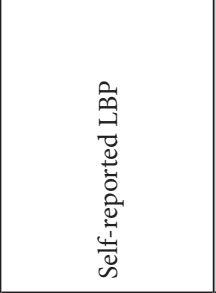 & 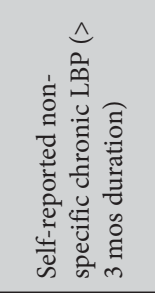 & 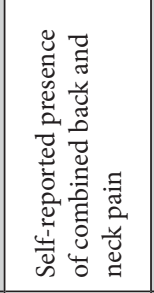 & 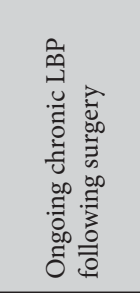 \\
\hline$=$ & $\infty$ & ঃ্ & $\vec{\lambda}$ & in & ల్ల & 尽 & $\infty$ & iे & $a$ \\
\hline & 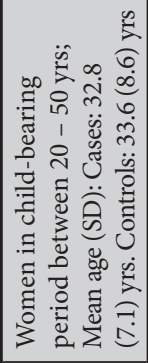 & 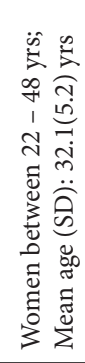 & 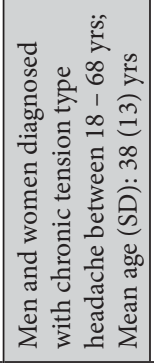 & 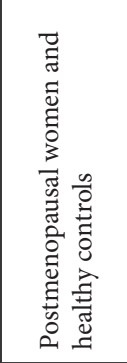 & 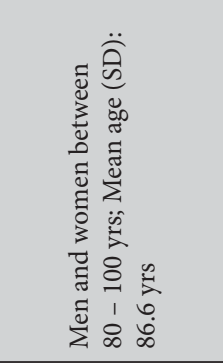 & 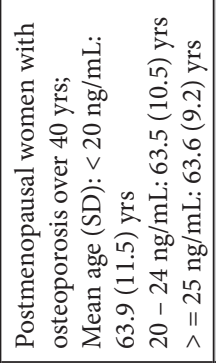 & 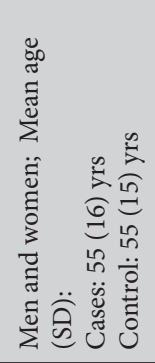 & 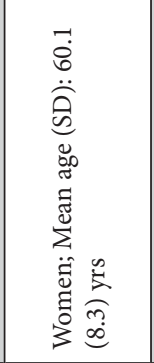 & 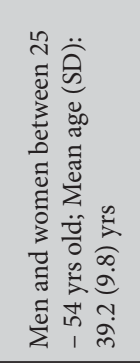 \\
\hline 苞 & 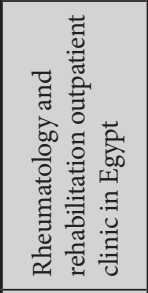 & 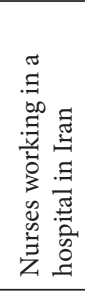 & 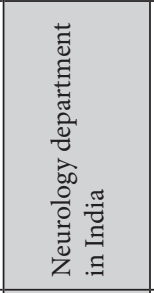 & 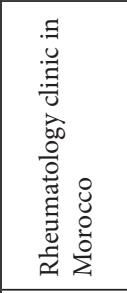 & 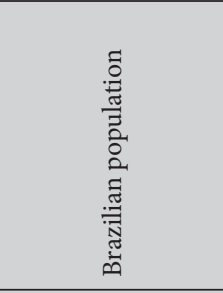 & 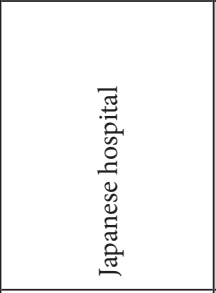 & 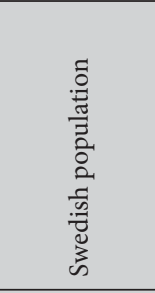 & 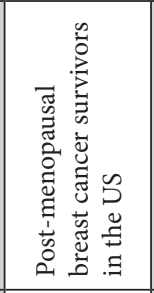 & 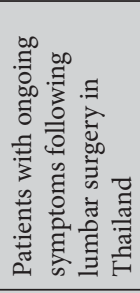 \\
\hline 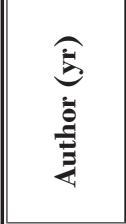 & 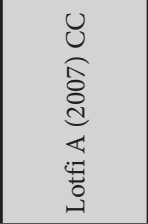 & 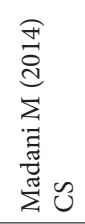 & 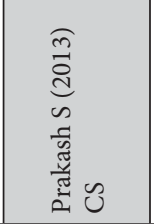 & 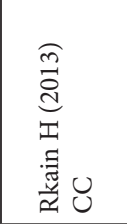 & 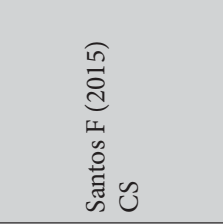 & 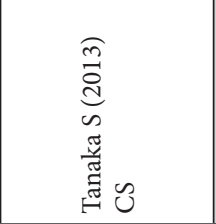 & 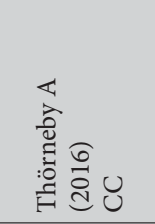 & 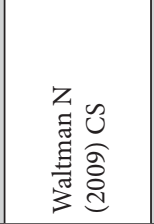 & 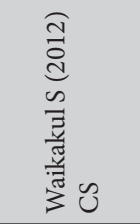 \\
\hline
\end{tabular}

www.painphysicianjournal.com 


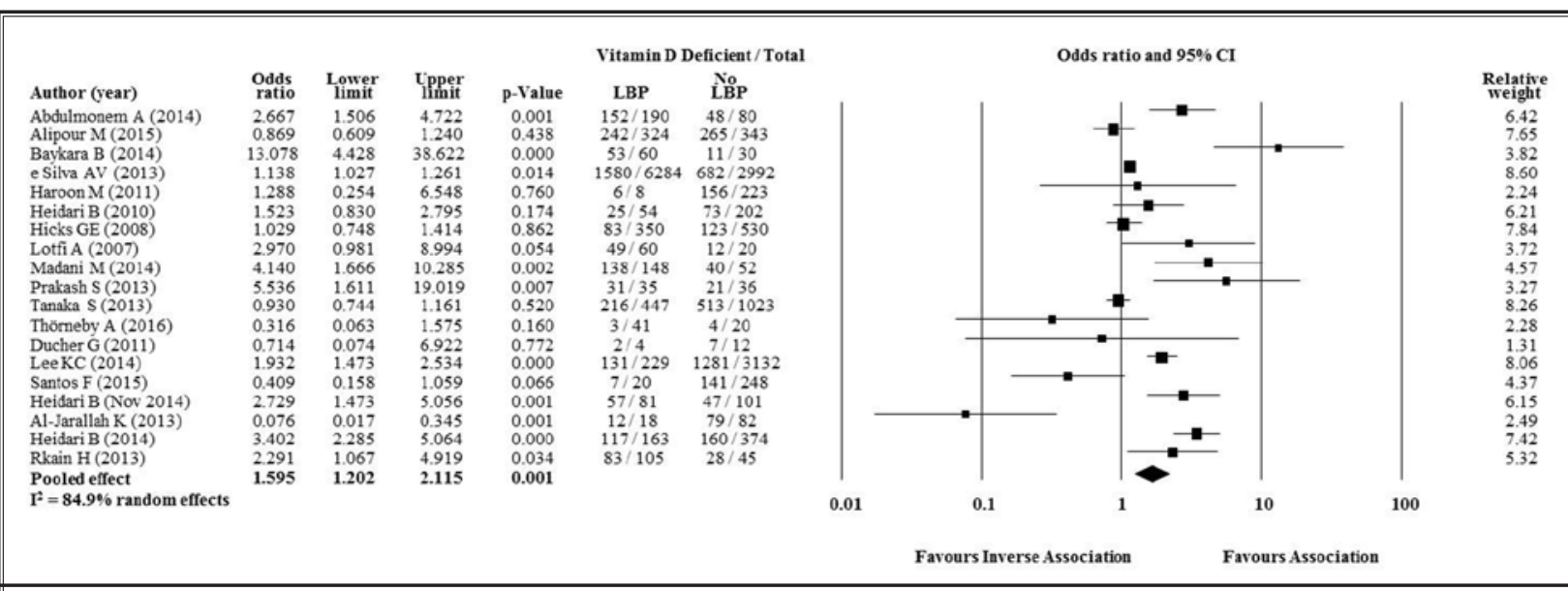

Fig. 2. Pooled odds ratio (95\% CI) for the association between vitamin D deficiency and LBP for all of the included studies. $\mathrm{LBP}=$ low back pain; $\mathrm{CI}=$ confidence interval

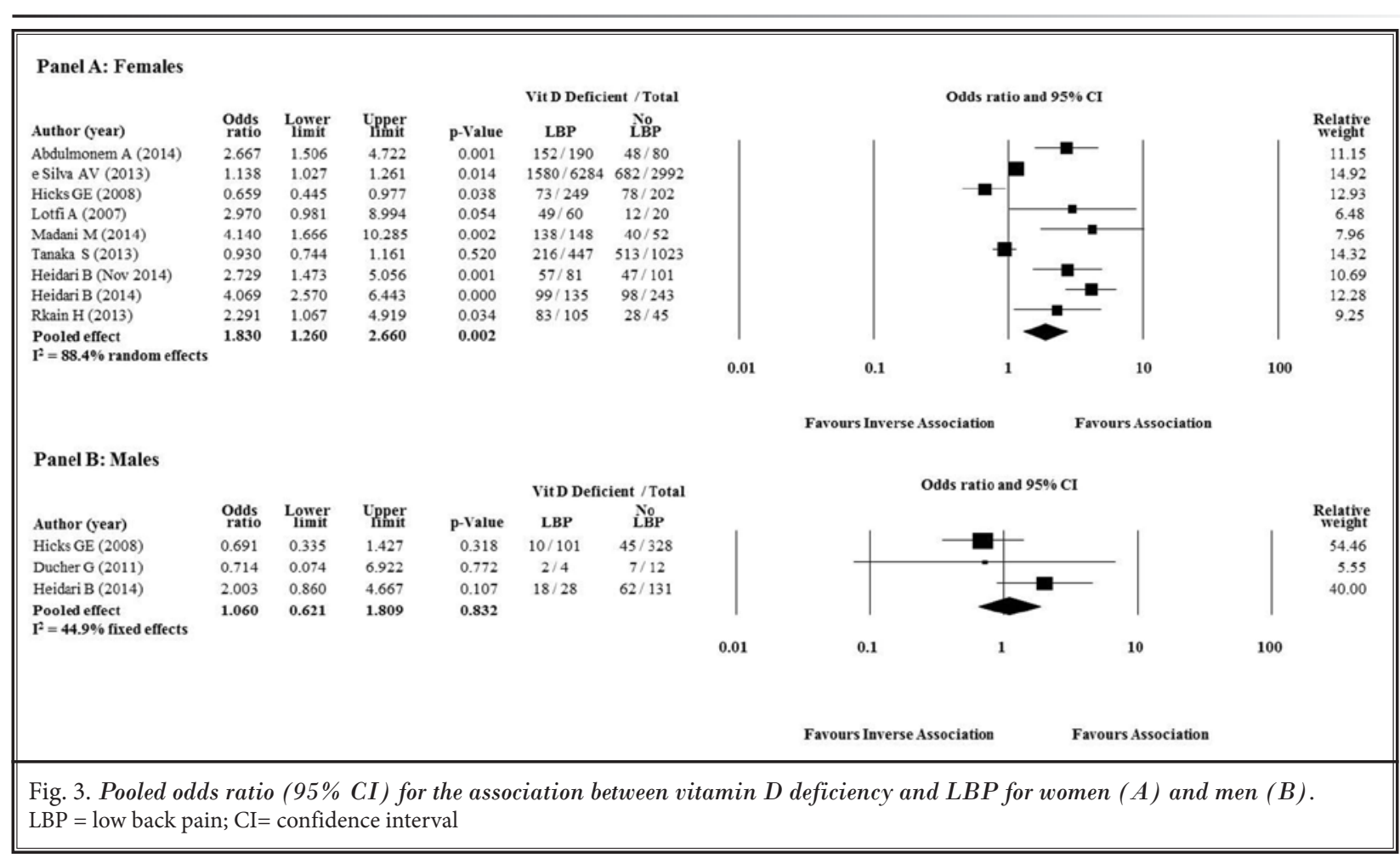

\section{Association between Vitamin D Deficiency and LBP}

The pooled results from 19 studies (11 cross-sectional studies and 8 case-control studies) demonstrated a significant association between vitamin $D$ deficiency and LBP (pooled OR $=1.60,95 \% \mathrm{Cl}: 1.20-2.12, P=$ $0.001, n=19$ ) (Fig. 2), where ' $n$ ' is the number of studies and an OR $>1$ indicates patients with LBP are more likely to have vitamin $D$ deficiency compared to those without LBP. This association was stronger for women (pooled OR $=1.83,95 \% \mathrm{Cl}: 1.26-2.66, P=0.002, \mathrm{n}=9$ ), but failed to be statistically significant for men (pooled $\mathrm{OR}=1.06,95 \% \mathrm{Cl}: 0.62-1.81, P=0.832, \mathrm{n}=3$ ) (Fig. 3). We further stratified our meta-analyses by age and cut-offs for vitamin $D$ deficiency.

The age of the patients varied substantially between 


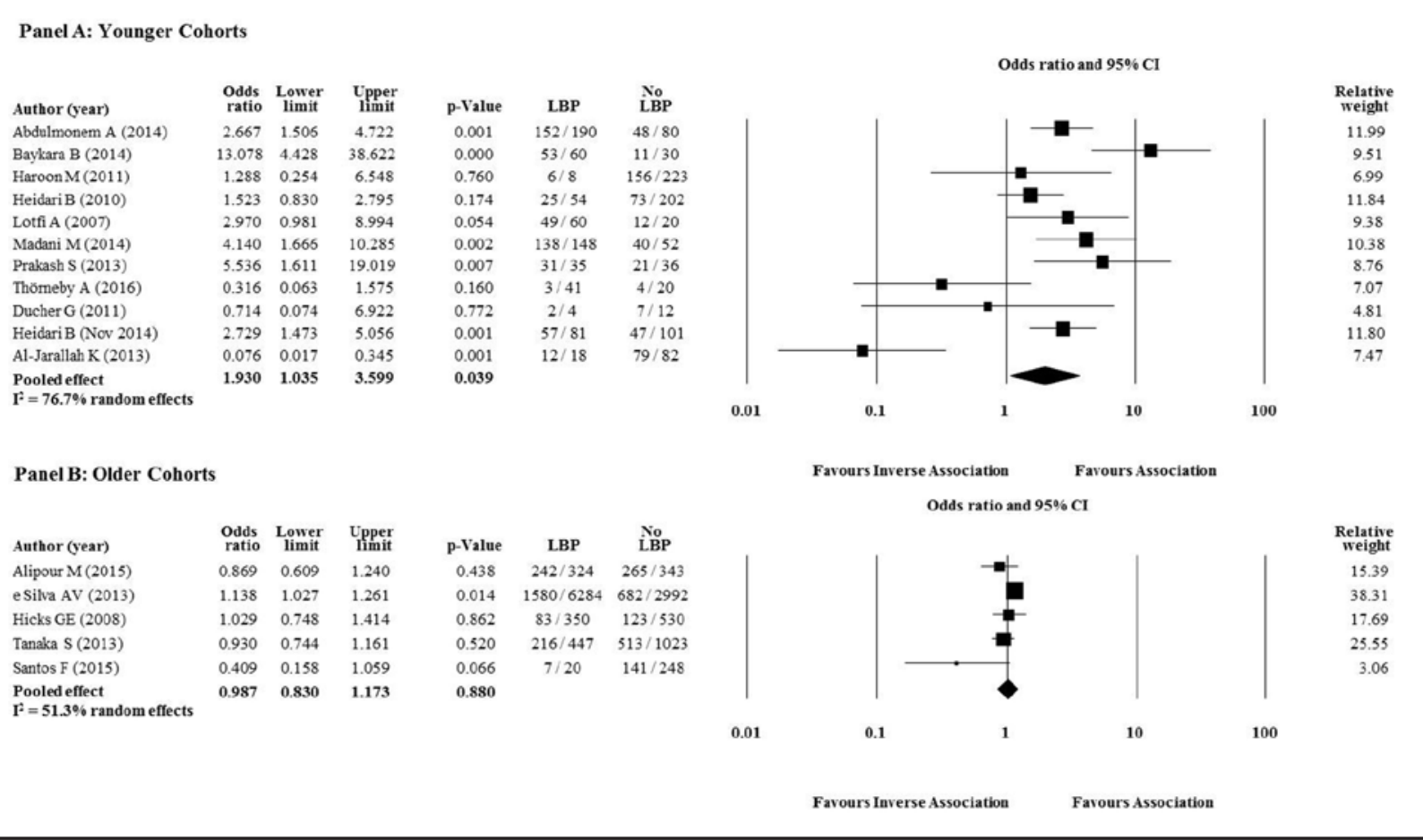

Fig. 4. Pooled odds ratio (95\% CI) for the association between vitamin D deficiency and LBP in cohorts with a mean age $<60$ years $(A)$ and $>60$ years $(B)$.

$\mathrm{LBP}=$ low back pain; $\mathrm{CI}=$ confidence interval

studies so we stratified our meta-analyses by samples with a mean age $<60$ or $>60$ years old to ensure the majority of postmenopausal women were included in the same category. There was a significant association between vitamin D deficiency and LBP in studies with a mean age $<60$ years old (pooled $\mathrm{OR}=1.93,95 \% \mathrm{Cl}$ : $1.04-3.60, P=0.039, \mathrm{n}=11$ ), but no association in the samples with a mean age $>60$ years old (pooled OR = $0.99,95 \% \mathrm{Cl}: 0.83-1.17, P=0.880, \mathrm{n}=5$ ) (Fig. 4). There was a strong association between vitamin $D$ deficiency and LBP in women $<60$ years old (pooled OR $=2.91,95 \%$ $\mathrm{Cl}: 2.03-4.17, P<0.001, \mathrm{n}=4)$, but no association in women $>60$ years old (pooled OR $=0.94,95 \% \mathrm{Cl}: 0.72$ $-1.22, P=0.631, \mathrm{n}=3$ ) (Fig. 5). The association between vitamin $D$ deficiency and LBP was investigated in men between $65-102$ years old in one study (OR $=0.69,95 \%$ $\mathrm{Cl}: 0.34-1.43, P=0.318)(21)$ and in men between $10-$ 19 years old in another study (OR $=3.67,95 \% \mathrm{Cl}: 0.17$ - 77.56, $P=0.404$ ) (Table 2) (42). Neither of these studies showed statistically significant results.

The cut-offs for vitamin D deficiency varied across studies and we stratified our meta-analyses accordingly. The pooled results from 13 studies that defined deficiency as having serum concentrations of 25(OH)D $<20 \mathrm{ng} / \mathrm{mL}$ (one study used $<21 \mathrm{ng} / \mathrm{mL}$ ) demonstrated no association between vitamin $D$ deficiency and LBP (pooled OR $=1.25,95 \% \mathrm{Cl}: 0.90-1.76, P=0.191, \mathrm{n}=$ $13)$, while the 7 studies that used $<10 \mathrm{ng} / \mathrm{mL}$ as a cut-off ( 2 studies used $<12 \mathrm{ng} / \mathrm{mL}(43,44)$ ) showed a significant association (pooled OR $=2.08,95 \% \mathrm{Cl}$ : $1.19-3.64, P=$ $0.010, n=7$ ) (Fig. 6).

\section{Association between Serum 25(OH)D and LBP}

There were 12 studies (4 cross-sectional and 8 case-control studies) that investigated the association between serum concentrations of 25(OH)D (continuous measure) and LBP. The pooled results from all of the studies (Fig. 7) and studies investigating women (Fig. 7) and patients $<60$ years old (Fig. 8) were similar to the findings for the association between vitamin $D$ deficiency and LBP. There were not enough data to pool the results for men or individuals $>60$ years old.

\section{Association between Vitamin D Deficiency and Pain Intensity}

There was a significant association between vita- 


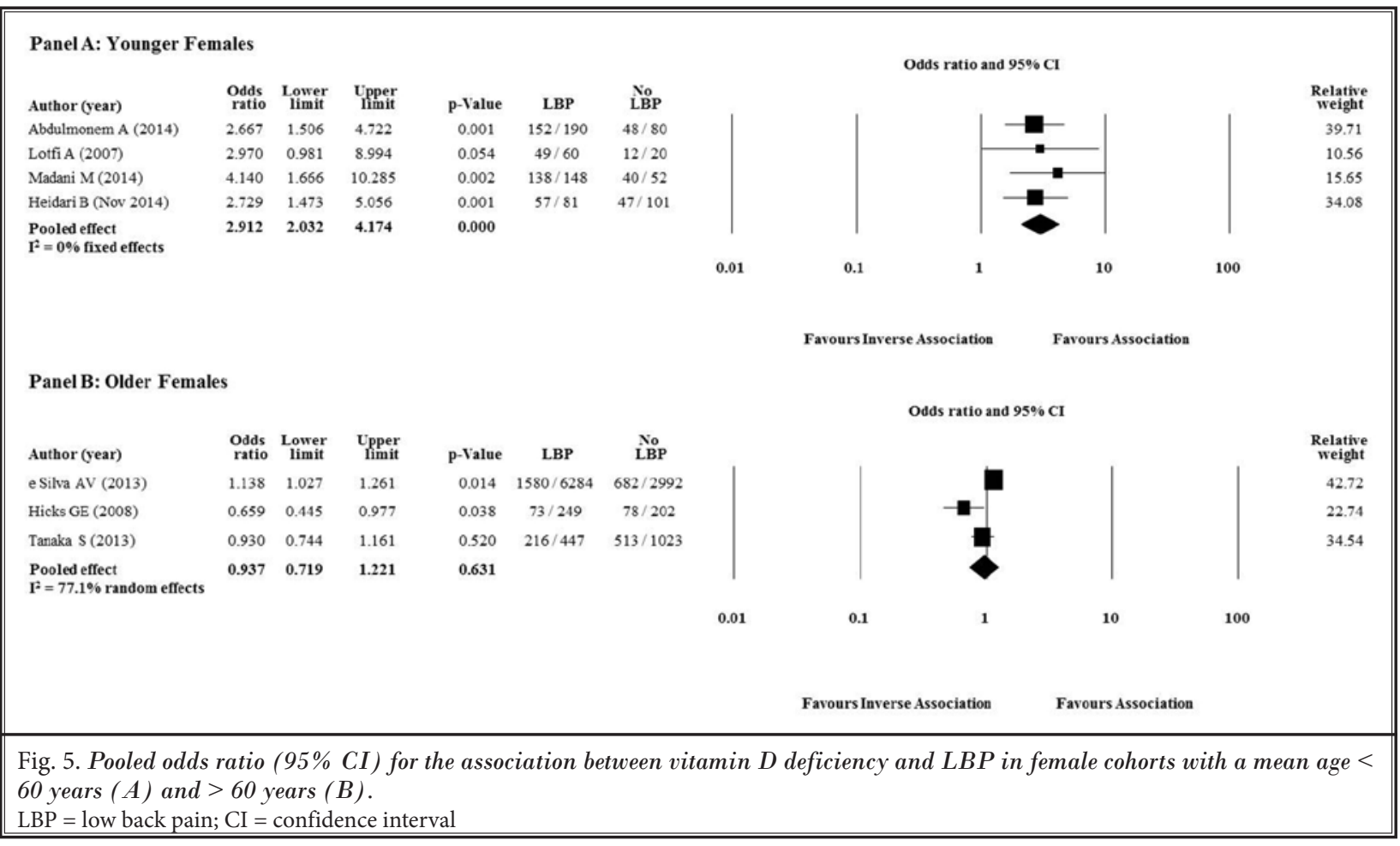

min $D$ deficiency and severe pain (compared to mild pain) (pooled OR $=1.98,95 \% \mathrm{Cl}: 1.05-3.75, P=0.036$, $\mathrm{n}=3$ ) (Fig. 9) but no association between pain intensity (continuous measure: $0-100$ scale) and vitamin $D$ deficiency (weighted $\mathrm{MD}=0.29,95 \% \mathrm{Cl}:-0.35-0.94$, $P=0.373, \mathrm{n}=4$ ) (Fig. 10). In addition, one conference abstract reported similar pain scores in people with deficient, insufficient, and normal vitamin $D$ levels, although no objective data was presented (45) (Table 2).

\section{Association between Serum 25(OH)D and Pain Intensity}

Our pooled results showed no association between serum 25(OH)D and pain intensity $(0-10$ scale) (pooled $\mathrm{r}=-0.02,95 \% \mathrm{Cl}:-0.21-0.17, P=0.812, \mathrm{n}=2$ ) (Fig. 11). In addition, one study failed to find a significant association between serum 25(OH)D and pain intensity (46), while another study found a significant association (35) (Table 2). Unfortunately, these studies failed to report objective data and were not included in the above meta-analysis. One cross-sectional study failed to find an association between serum 25(OH)D and severe pain (47), while another study reported a significant negative correlation between serum 25(OH)D and LBP (48) (Table 2). However, it was not clear how the latter study assessed pain intensity or the presence of LBP.

\section{Discussion}

This is the first systematic review to investigate the association between vitamin D and LBP and the association between vitamin $D$ and pain intensity in patients with LBP, which is particularly important given the increasing interest in vitamin $D$ supplementation for the management of $\operatorname{LBP}(28,29)$. Our results showed that patients with LBP are more likely to have vitamin D deficiency (particularly severe deficiency) and lower serum concentrations of $25(\mathrm{OH}) \mathrm{D}$, compared to those without LBP. The relationship between vitamin $D$ deficiency and LBP is stronger in women and in those $<60$ years old. On the other hand, there is conflicting evidence that vitamin $D$ deficiency influences pain intensity in patients with LBP.

\section{Association between Vitamin D and LBP}

The results of this review suggest the association between vitamin D deficiency and LBP is influenced by age and gender, with stronger associations observed in younger women ( $<60$ years old). However, the geographical location of the included studies may partially explain these results; therefore, we conducted a number of sensitivity analyses to explore this issue. There was a strong association between vitamin $D$ deficiency 
Pain Physician: November/December 2017: 20: 611-640

\begin{tabular}{|c|c|c|c|c|c|c|c|c|c|}
\hline & 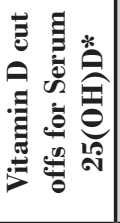 & 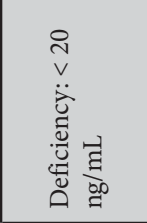 & 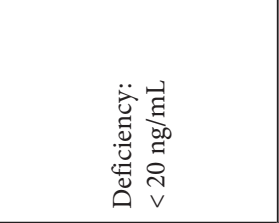 & 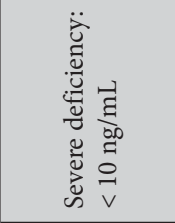 & 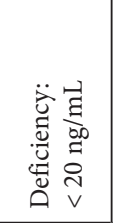 & 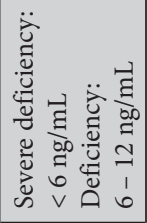 & 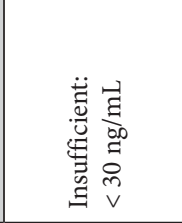 & 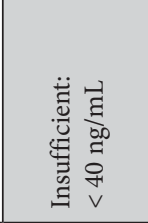 & 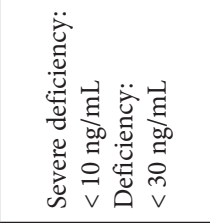 \\
\hline & 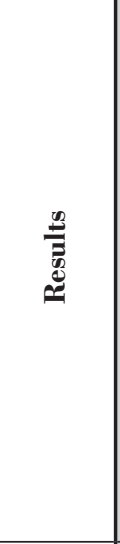 & 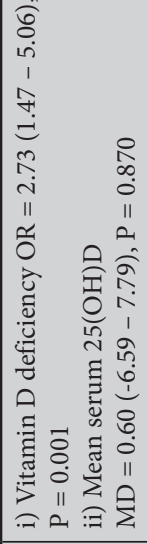 & 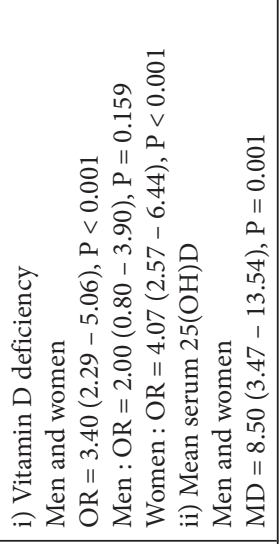 & 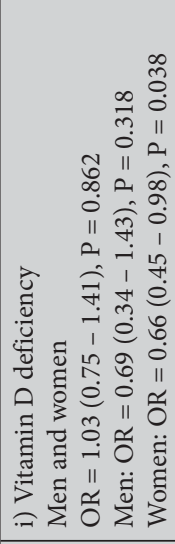 & 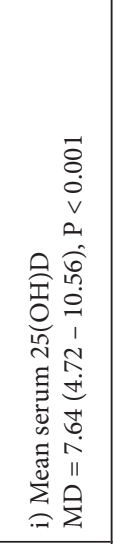 & 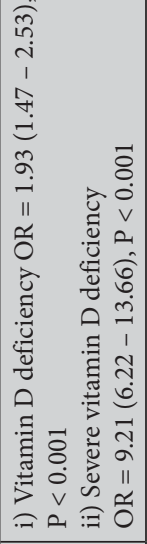 & 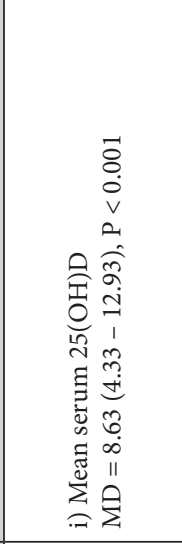 & 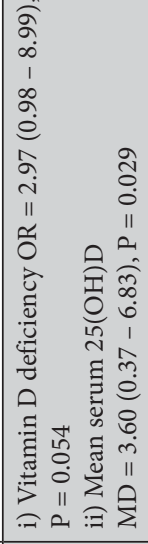 & 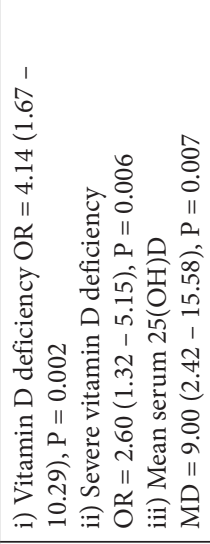 \\
\hline & 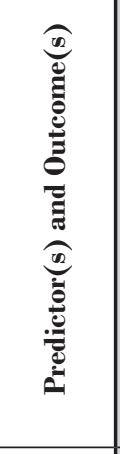 & 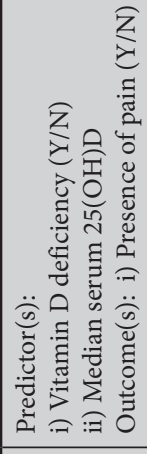 & 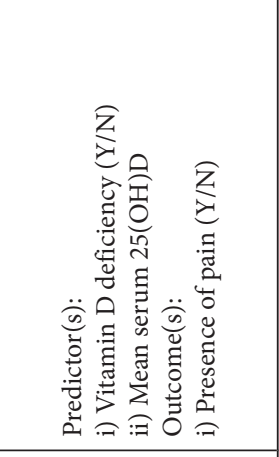 & 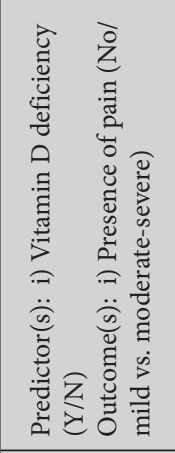 & 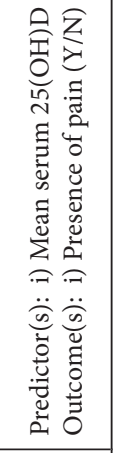 & 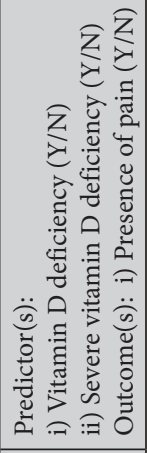 & 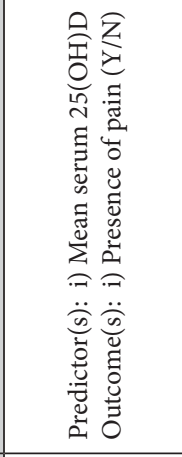 & 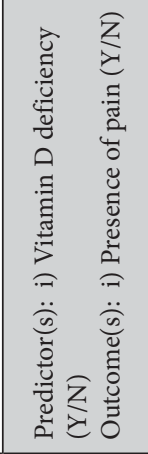 & 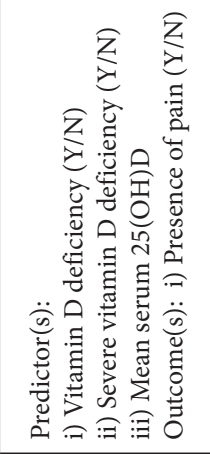 \\
\hline 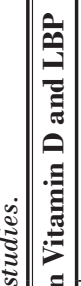 & 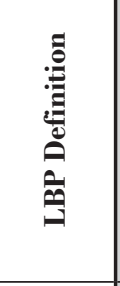 & 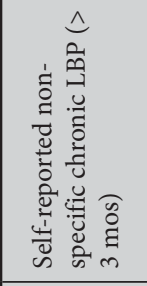 & 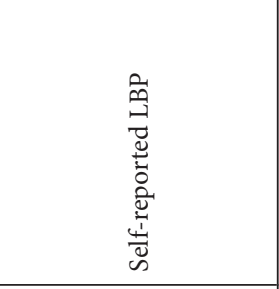 & 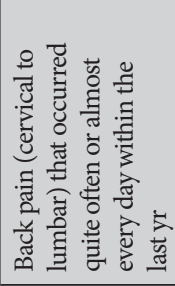 & 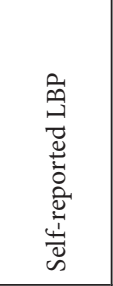 & 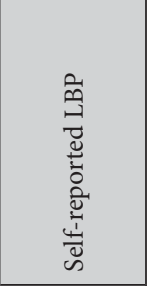 & 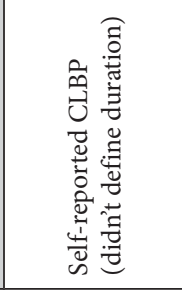 & 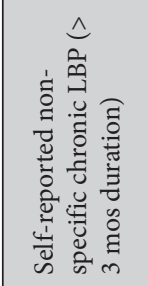 & 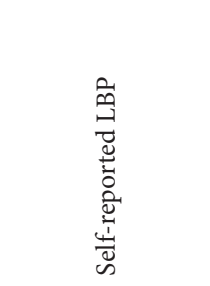 \\
\hline$\frac{\pi}{0}$ & $\Rightarrow$ & $\stackrel{\sim}{-\infty}$ & $\stackrel{\mathscr{I}}{\mathscr{I}}$ & ณू & สิ & 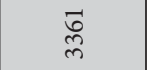 & ஓ̊̀ & 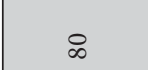 & ષ્సి \\
\hline 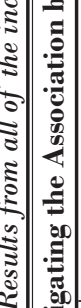 & 离 & 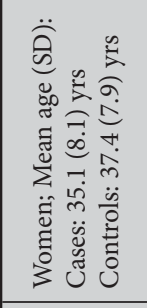 & 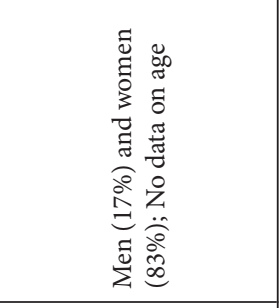 & 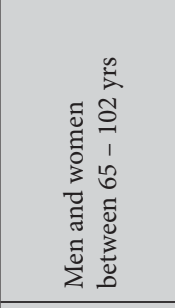 & 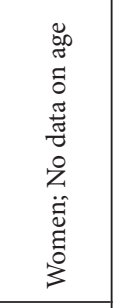 & 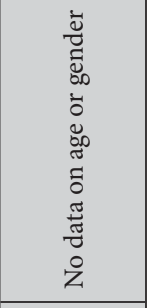 & 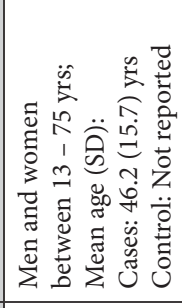 & 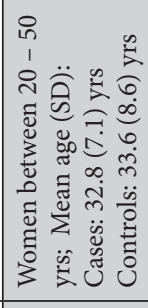 & 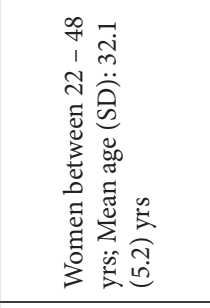 \\
\hline 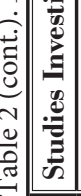 & 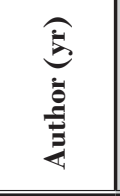 & 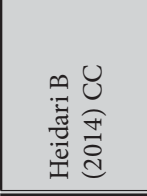 & 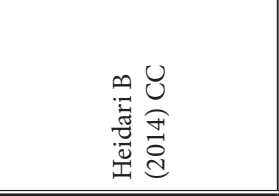 & 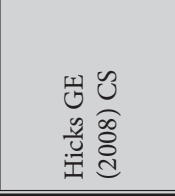 & 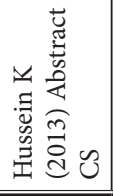 & 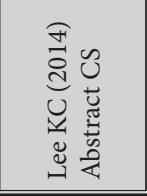 & 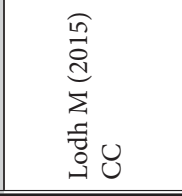 & 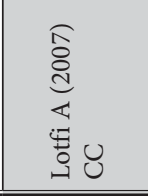 & 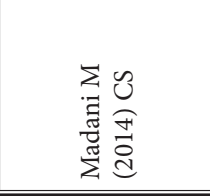 \\
\hline
\end{tabular}


Vitamin D and Low Back Pain

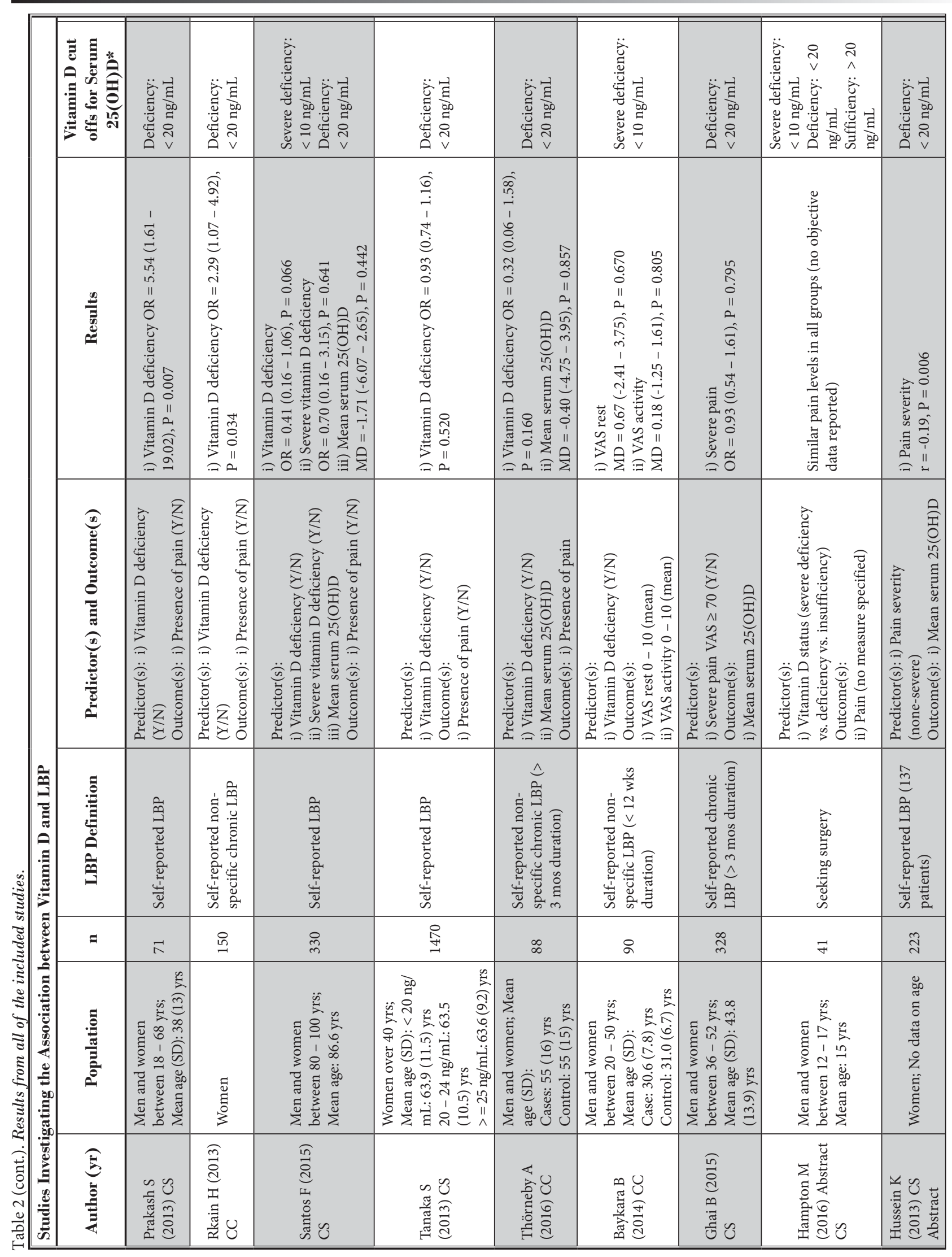

www.painphysicianjournal.com 
Pain Physician: November/December 2017: 20: 611-640

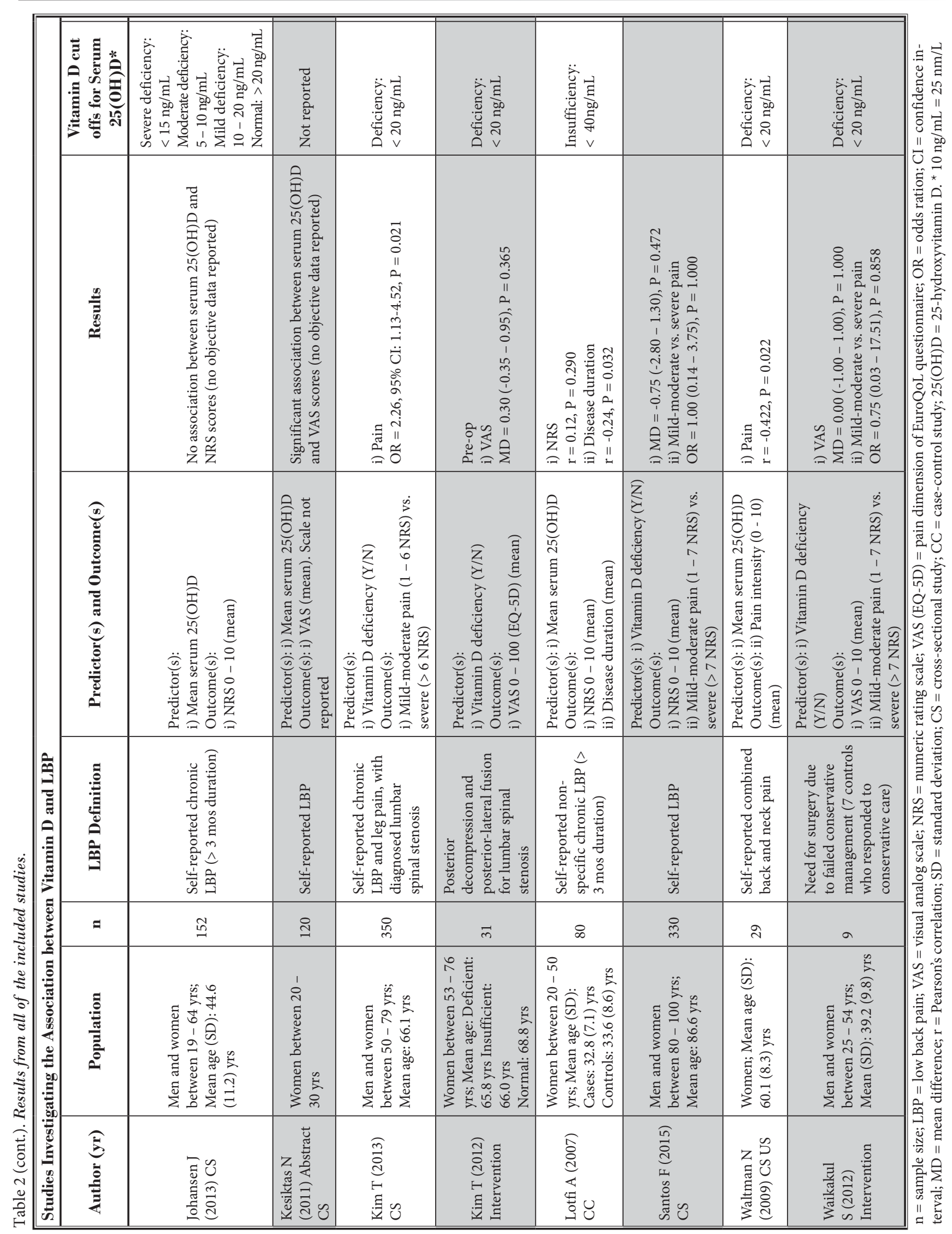


and LBP when we pooled the 10 studies conducted in the Middle-East/Mediterranean region, particularly in female-only samples (Appendix 3). The one study conducted in this region investigating a male-only sample found no association (Table 2) (37). In addition, there was no association between vitamin $D$ deficiency and LBP when pooling the 9 studies conducted outside of the Middle-East/Mediterranean region (Appendix 4). Since all of the studies investigating the association between vitamin D deficiency and LBP in younger women were conducted in the Middle-East/Mediterranean region, this may partially account for the strong associations found in this population given the climatic and cultural factors (e.g., sun exposure, cultural veiling, physical activity levels, obesity) likely to confound the relationship between vitamin $D$ deficiency and LBP. Furthermore, all of the studies investigating the association between vitamin D deficiency and LBP in women $>60$ years old were conducted outside of the Middle-East/Mediterranean region, which might ex- plain the lack of association found in this population. A high prevalence of vitamin D deficiency in this age group may also explain the lack of association (49), as differences in vitamin $D$ status between those with and without LBP would be negligible (40). However, we did not observe a trend suggesting lower baseline vitamin D levels in studies with older samples (Table 1).

Another explanation for the significant association between vitamin D and LBP in women could be related to a higher number of studies including women (women: $n=13$; men: $n=4$ ). This may be the result of a higher prevalence of LBP (49) and vitamin D deficiency (50) in this population, although additional hypotheses deserve attention. It is well-established that vitamin D can facilitate the uptake of calcium and lead to bone mineralisation (51), which is particularly important for women where age and hormonal-related bone density loss (52) can increase the risk of osteoporosis (53), potentially resulting in pain. Therefore, investigating the associations between vitamin D deficiency and health

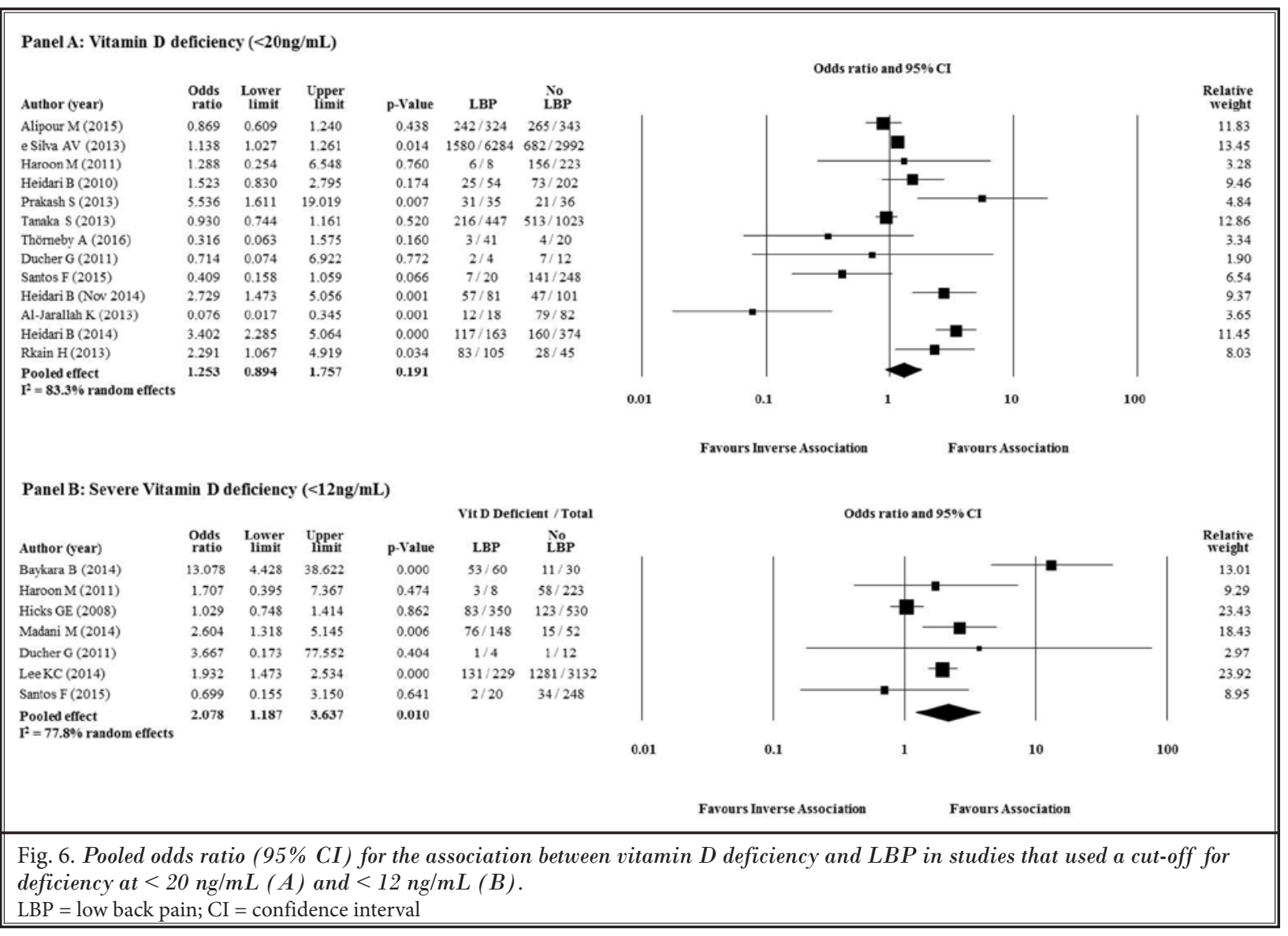




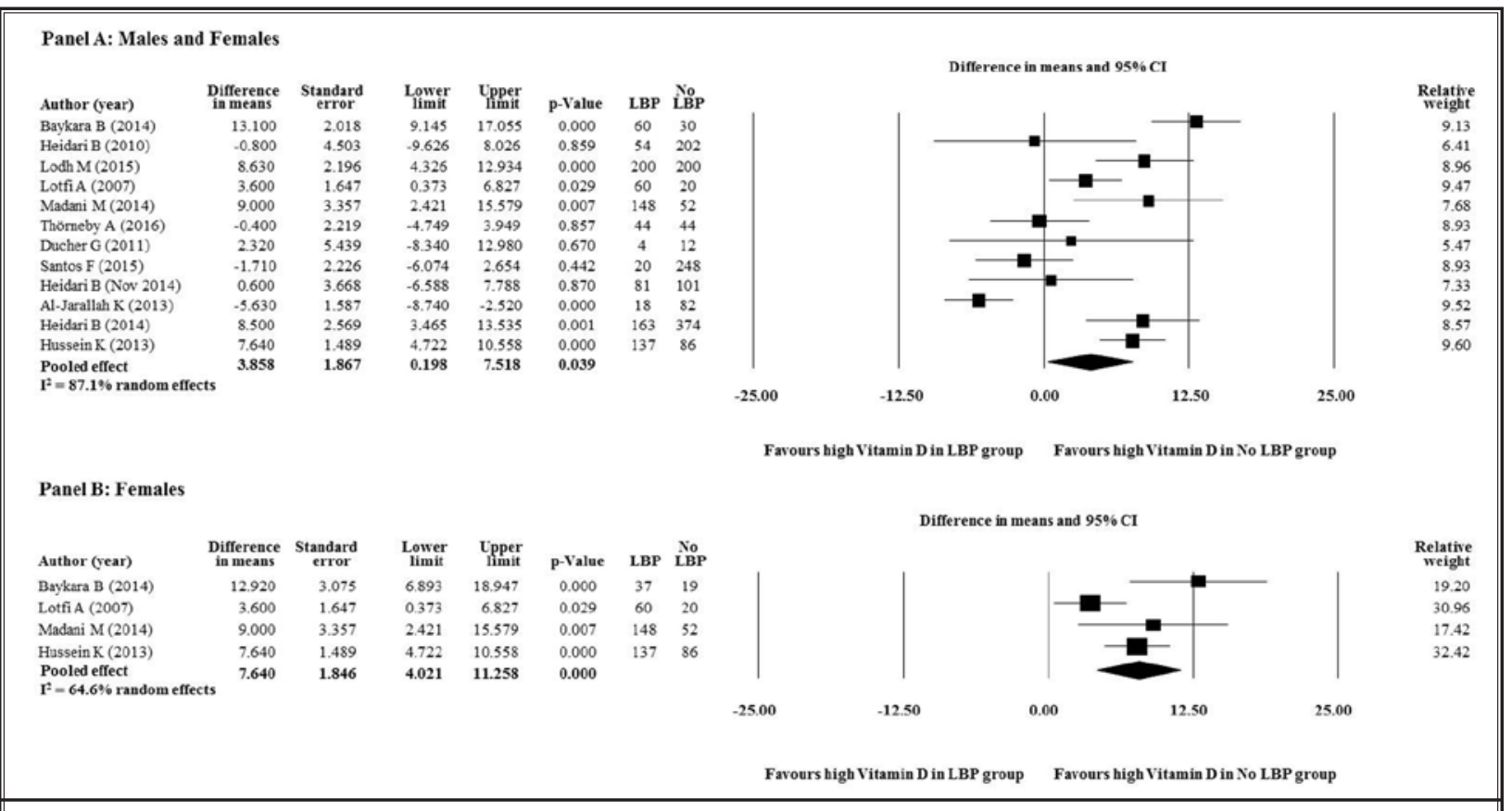

Fig. 7. Pooled odds ratio (95\% CI) for the association between association between serum concentrations of $25(\mathrm{OH}) \mathrm{D}$ and LBP for all of the included studies $(A)$ and for women $(B)$.

$\mathrm{LBP}=$ low back pain; $\mathrm{CI}=$ confidence interval

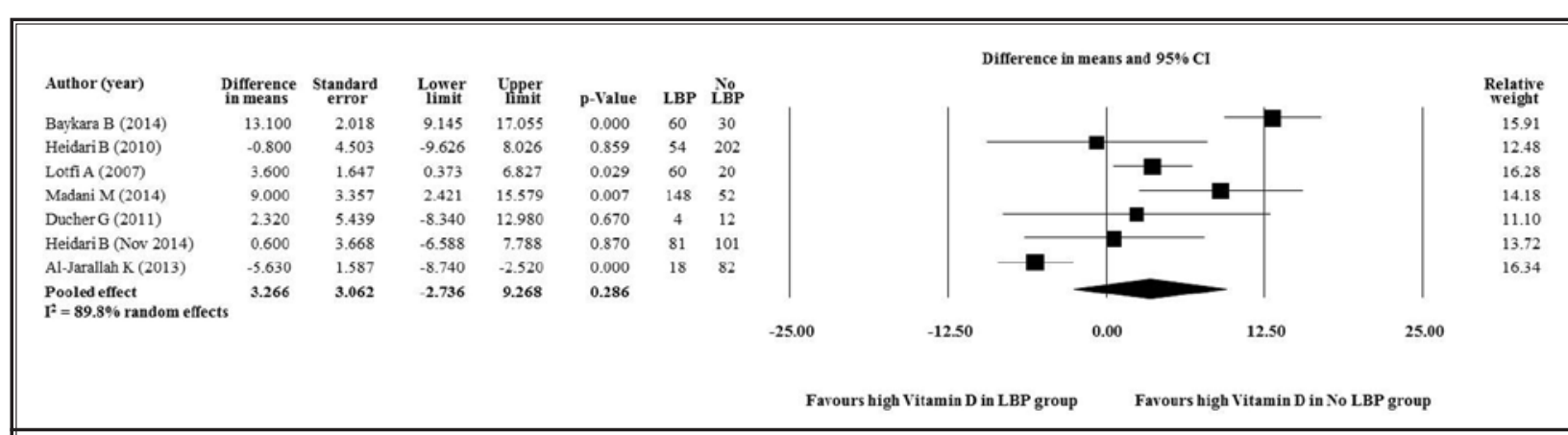

Fig. 8. Pooled odds ratio (95\% CI) for the association between serum concentrations of $25(\mathrm{OH}) \mathrm{D}$ and LBP in cohorts with a mean age $<60$ years.

$\mathrm{LBP}=$ low back pain; $\mathrm{CI}=$ confidence interval

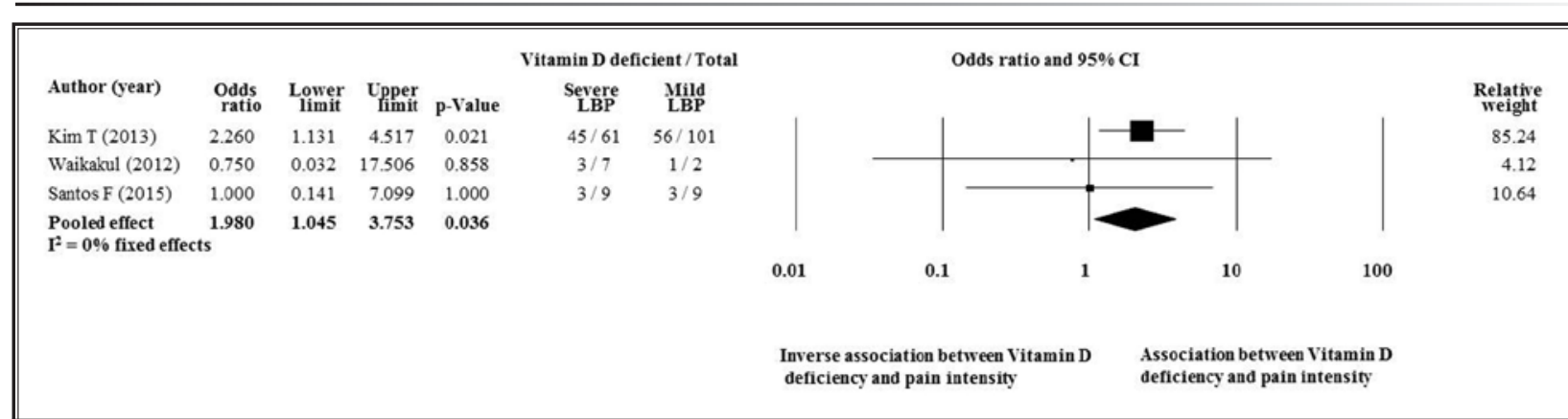

Fig. 9. Weighted mean difference (95\% CI) for the association between vitamin D deficiency and pain intensity (mild vs. severe). $\mathrm{LBP}=$ low back pain; $\mathrm{CI}=$ confidence interval 


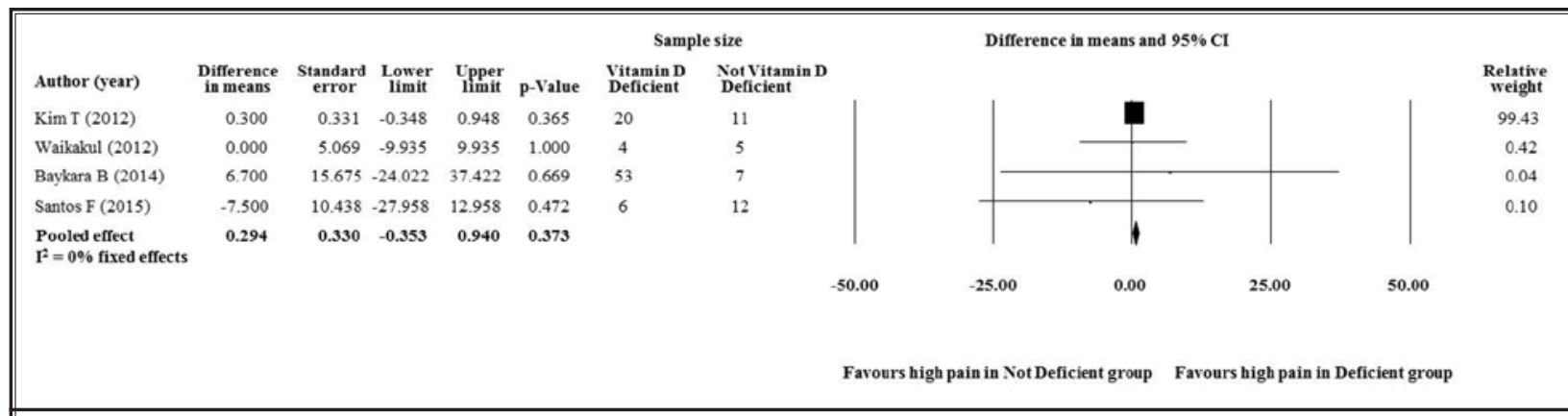

Fig. 10. Weighted mean difference (95\% CI) for the association between mean pain intensity and vitamin $D$ deficiency. $\mathrm{CI}=$ confidence interval

\begin{tabular}{|c|c|c|c|c|c|c|c|c|c|c|}
\hline \multirow[b]{2}{*}{ Author (year) } & \multirow[b]{2}{*}{ Correlation } & \multirow[b]{2}{*}{$\begin{array}{c}\text { Lower } \\
\text { limit }\end{array}$} & \multirow[b]{2}{*}{$\begin{array}{c}\text { Upper } \\
\text { limit }\end{array}$} & \multicolumn{7}{|c|}{ Correlation and $95 \% \mathrm{CI}$} \\
\hline & & & & p-Value & $\begin{array}{l}\text { Sample } \\
\text { size }\end{array}$ & & & & & $\begin{array}{c}\text { Relative } \\
\text { weight }\end{array}$ \\
\hline Waltman N (2009) & -0.422 & -0.683 & -0.066 & 0.022 & 29 & 1 & & & & 25.24 \\
\hline Lotfi A (2007) & 0.120 & -0.102 & 0.331 & 0.290 & 80 & & & & & 74.76 \\
\hline \multirow[t]{2}{*}{$\begin{array}{l}\text { Pooled effect } \\
\mathrm{r}^{2}=84.2 \% \text { random effects }\end{array}$} & ts $\quad-0.023$ & -0.213 & 0.168 & 0.812 & & & & & & \\
\hline & & & & & -1.00 & -0.50 & 0.00 & 0.50 & 1.00 & \\
\hline
\end{tabular}

conditions (such as LBP) in women may be considered a research priority and explain the higher number of studies in women. A limited number of studies in men have resulted in imprecise pooled estimates of association. Therefore, a positive association between vitamin $D$ and LBP in men should not be ruled out and needs confirmation in larger samples. Nevertheless, the findings of our study demonstrate an increased prevalence of vitamin D deficiency in younger women with LBP. This may provide a rationale for targeting vitamin $D$ supplementation for the management of LBP in this population or considering vitamin D supplementation to reduce the risk of developing LBP.

The presentation or chronicity of LBP may also be important to consider when determining which populations with LBP are at greatest risk of vitamin D deficiency. To explore this we conducted a number of sensitivity analyses. We found no association between vitamin D deficiency and chronic LBP (Appendix 5) or between vitamin $D$ deficiency and LBP resulting from osteoporosis or low bone mass (Appendix 6) when pooling all of the available studies. However, there was a strong association between vitamin $D$ deficiency and chronic LBP in women (Appendix 5), although the strength of this association may be explained by the studies' geographical location, as all the studies were conducted in the Middle-East/Mediterranean region. Furthermore, the lack of association between vitamin $D$ deficiency and LBP resulting from osteoporosis or low bone mass may have been due to a small number of studies investigating this population $(n=2)$.

Our results point towards the importance of considering the degree of vitamin $D$ deficiency in people with LBP since this association strengthened when we only considered studies that used a cut-off of $<10-12$ $\mathrm{ng} / \mathrm{mL} 25(\mathrm{OH}) \mathrm{D}$. This highlights a stronger association between LBP and severe vitamin D deficiency and is consistent with the findings of another review that showed severe deficiencies were more common in individuals with chronic widespread pain compared to individuals without these symptoms (15). This may highlight the importance of screening for severe vitamin D deficiencies in these populations to potentially reduce the risk of serious disease (54-56).

\section{Association between Vitamin D and Pain Intensity}

Understanding how vitamin D deficiency influences pain intensity may provide insight into the potential role of vitamin D supplementation for the man- 
agement of LBP. However, substantial heterogeneity between studies investigating the association between vitamin $D$ and pain intensity makes it hard to draw firm conclusions about the role vitamin D plays in patients already suffering from LBP. We were only able to pool results from 4 studies, and the findings appear to be dictated by how the variables were analyzed. There was a significant association between mean vitamin D levels (continuous) and severe pain (dichotomous), but there was no association between vitamin D deficiency (dichotomous) and pain intensity (continuous). To add to these conflicting findings, some individual studies failed to find an association between serum 25(OH)D and pain intensity $(46,47)$, while others found a significant association between serum 25(OH)D and duration of pain (17). Given the small number of studies investigating the association between vitamin $D$ and pain intensity, we could not identify a trend between positive findings and study characteristics (e.g., gender, age, geographical location).

Given these conflicting findings, it is important to carefully consider the rationale and current evidence on vitamin D supplementation for LBP before additional studies are implemented. Research suggests that vitamin $D$ levels influence the presence of inflammatory markers $(22,25-27)$ and can modulate sensory neuron excitability $(23,24)$. In addition, the influence of vitamin $D$ levels on muscle strength has been highlighted $(57,58)$ and may provide an explanation for the association between vitamin D deficiency and LBP and a rationale for using vitamin $D$ supplementation for treatment. However, evidence supporting the relationship between vitamin D deficiency and reduced muscle strength is conflicting (59-62), and even if vitamin D deficiency could be regarded as a predictor of muscle weakness, muscle weakness is not consistently associated with the prevalence or risk of developing LBP (63$65)$, nor do improvements in muscle strength correlate with treatment outcomes (66). There has already been a number of studies conducted investigating vitamin D supplementation for the management of non-specific LBP or LBP resulting from osteoporosis or vertebral fractures, however, the results are far from promising. Three randomized controlled trials $(28,67,68)$ failed to show that vitamin D3 was superior to a placebo for reducing pain intensity in individuals with non-specific LBP. This is despite the differences in their intervention dosage (10 - 179 ug per day) and duration (6 - 16 weeks) and despite the vitamin D3 groups achieving normal 25(OH)D concentrations post-intervention (>
$20 \mathrm{ng} / \mathrm{mL})(28,67)$. Similarly, vitamin D supplementation for the management of LBP resulting from osteoporosis or vertebral fractures has yielded disappointing results (69). Therefore, given the poor association between vitamin D and pain intensity in patients with LBP and the findings from existing clinical trials, further research is needed before vitamin D supplementation is recommended for the management of LBP.

\section{Strengths and Limitations}

This systematic review has numerous strengths. First, to get a comprehensive understanding of the relationship between vitamin D and LBP it was necessary to include different study designs in our review (cross-sectional, case-control, and case-series). In addition, although a number of studies (including abstracts) failed to publish adequate data for initial inclusion in our meta-analyses $(n=10)$, we contacted these authors and were able to obtain raw data from 5 studies which significantly strengthened the results of this review. Second, including conference abstracts and the abstracts of articles where the full text was not accessible reduced the risk of neglecting important data, while minimizing the risk of publication bias (70). Although the quality of these data is unknown, we conducted a number of sensitivity analyses, and the exclusion of studies where the full text was not accessible (including conference abstracts) did not influence the main findings of this review (Appendix 7).

This review has a number of limitations. First, most of the studies investigating the association between vitamin D and pain intensity in people with LBP used different statistical analyses and investigated different outcomes. This precluded the inclusion of all the data into one meta-analysis. Second, the majority of studies $(n=20)$ failed to adjust their findings for potential confounding variables (e.g., age, gender, sun exposure, skin type, use of supplementation, muscle strength), so we decided to use the unadjusted values in our metaanalyses for consistency and stratify our meta-analyses by age, gender, and cut-offs of vitamin D deficiency to investigate how these factors influence the relationship between vitamin D and LBP. Furthermore, we conducted a number of sensitivity analyses to investigate the influence of study geographical location and the presentation of LBP. Finally, there were no longitudinal studies investigating whether vitamin D deficiency increases the risk of developing LBP. Information from longitudinal studies is extremely valuable if vitamin D supplementation is to be considered a prevention strat- 
egy for LBP in the future since cross-sectional studies cannot infer the temporal relationship between vitamin D levels and LBP (causation).

\section{Conclusion}

Vitamin D deficiency is associated with LBP, with stronger associations observed in younger women and those with severe levels of deficiency. The association between vitamin $D$ levels and pain intensity is inconsistent. The findings from existing clinical trials do not support the use of vitamin $D$ supplementation for the management of LBP. However, the results of this review have furthered our understanding on which populations demonstrate the greatest degree of vitamin $D$ deficiency and may guide the implementation of future studies on vitamin D supplementation for LBP. In addition, although current evidence does not support the widespread screening of vitamin $D$ levels in patients with LBP, clinicians should certainly consider this course of action for populations at increased risk of vitamin D deficiency (i.e., younger women and patients with chronic symptoms), as it is inexpensive, safe, and might improve symptoms. Further research evaluating the assessment and treatment of vitamin D deficiency will clarify their role in this difficult therapeutic area. Finally, longitudinal studies investigating whether vitamin D deficiency increases the risk of developing LBP are needed to determine the potential role of vitamin $D$ in the prevention of LBP. 
Appendix 1. Search strategy.

\begin{tabular}{|c|c|}
\hline MEDLINE & Searches \\
\hline Vitamin D & $\begin{array}{l}\text { exp vitamin D/ } \\
\text { "vitamin D".mp } \\
\text { "vitamin D2".mp } \\
\text { "vitamin D3".mp } \\
\text { "1-alpha hydroxyvitamin D3".mp } \\
\text { "1-alpha hydroxycalciferol".mp } \\
\text { "1,25 dihydroxyvitamin D3".mp } \\
\text { "1,25 dihydroxycholecalciferol".mp } \\
\text { " } 25 \text { hydroxycholecalciferol".mp } \\
\text { " } 25 \text { hydroxyvitamin D".mp } \\
\text { "alfacalcidol".mp } \\
\text { "calcidiol".mp } \\
\text { "calcitriol".mp } \\
\text { "calcifediol".mp } \\
\text { "calciferol".mp } \\
\text { "ergocalciferol".mp } \\
\text { exp Ergocalciferols/ } \\
\text { "cholecalciferol".mp } \\
\text { exp Cholecalciferol/ } \\
1 \text { or } 2 \text { or } 3 \text { or } 4 \text { or } 5 \text { or } 6 \text { or } 7 \text { or } 8 \text { or } 9 \text { or } 10 \text { or } 11 \text { or } 12 \\
\text { or } 13 \text { or } 14 \text { or } 15 \text { or } 16 \text { or } 17 \text { or } 18 \text { or } 19\end{array}$ \\
\hline \multirow[t]{2}{*}{ Low back pain } & $\begin{array}{l}\text { exp Back Pain/ } \\
\text { "back pain".mp } \\
\text { "backpain".mp } \\
\text { exp Low Back Pain/ } \\
\text { "low back pain".mp } \\
\text { "backache".mp } \\
\text { "back ache".mp } \\
\text { (lumbar adj5 pain).ti,ab } \\
\text { "lumbar pain".mp } \\
\text { "spinal pain".mp } \\
\text { "lumbago".mp. } \\
\text { "lower back pain".mp } \\
\text { "dorsalgia".mp } \\
\text { "vertebral pain".mp } \\
21 \text { or } 22 \text { or } 23 \text { or } 24 \text { or } 25 \text { or } 26 \text { or } 27 \text { or } 28 \text { or } 29 \text { or } 30 \\
\text { or } 31 \text { or } 32 \text { or } 33 \text { or } 34\end{array}$ \\
\hline & $\begin{array}{l}20 \text { and } 35 \\
\text { Limit } 36 \text { to humans }\end{array}$ \\
\hline CINHAL & Searches \\
\hline Vitamin D & $\begin{array}{l}\text { MH "Vitamin D+" } \\
\text { MH "Vitamin D Deficiency+" } \\
\text { MH "Ergocalciferols" } \\
\text { MH "Cholecalciferol" } \\
\text { "vitamin D" } \\
\text { "vitamin D2" } \\
\text { "vitamin D3" } \\
\text { "1-alpha hydroxyvitamin D3" } \\
\text { "1,25 dihydroxyvitamin D3" } \\
\text { "1,25 dihydroxycholecalciferol" } \\
\text { " } 25 \text {-hydroxycholecalciferol" } \\
\text { " } 25 \text { hydroxycholecalciferol" } \\
\text { " } 25 \text { hydroxyvitamin D" } \\
\text { " } 25 \text {-hydroxy-vitamin D" } \\
\text { "alfacalcidol" } \\
\text { "calcidiol" } \\
\text { "calcitriol" } \\
\text { MH "Calcitriol" } \\
\text { "calcifediol" } \\
\text { "calciferol" } \\
1 \text { or } 2 \text { or } 3 \text { or } 4 \text { or } 5 \text { or } 6 \text { or } 7 \text { or } 8 \text { or } 9 \text { or } 10 \text { or } 11 \text { or } 12 \\
\text { or } 13 \text { or } 14 \text { or } 15 \text { or } 16 \text { or } 17 \text { or } 18 \text { or } 19 \text { or } 20\end{array}$ \\
\hline
\end{tabular}

Appendix 1 (cont.). Search strategy.

\begin{tabular}{|c|c|}
\hline \multirow[t]{2}{*}{ Low back pain } & \begin{tabular}{|l} 
(MH "Back Pain+") \\
"back pain" \\
(MH "Low Back Pain") \\
"low back pain" \\
"lumbago"" \\
"backache" \\
"back ache" \\
"lumbar pain" \\
"spinal pain" \\
"backpain" \\
"lower back pain" \\
"dorsalgia" \\
"vertebral pain" \\
22 or 23 or 23 or 24 or 25 or 26 or 27 or 28 or 29 or 30 \\
or 31 or 32 or 33 or 34
\end{tabular} \\
\hline & 21 and 35 \\
\hline EMBASE & Searches \\
\hline Vitamin D & $\begin{array}{l}\text { exp vitamin D/ } \\
\text { "vitamin D".mp } \\
\text { "vitamin D2".mp } \\
\text { "vitamin D3".mp } \\
\text { "1-alpha hydroxyvitamin D3".mp } \\
\text { "1-alpha-hydroxy-calciferol".mp } \\
\text { "1,25 dihydroxyvitamin D3".mp } \\
\text { "1,25 dihydroxycholecalciferol".mp } \\
\text { "25 hydroxycholecalciferol".mp } \\
\text { "25 hydroxyvitamin D".mp } \\
\text { "alfacalcidol".mp } \\
\text { "calcidiol".mp } \\
\text { "calcitriol".mp } \\
\text { "calcifediol".mp } \\
\text { "calciferol".mp } \\
\text { "ergocalciferol".mp } \\
\text { "cholecalciferol".mp } \\
1 \text { or } 2 \text { or } 3 \text { or } 4 \text { or } 5 \text { or } 6 \text { or } 7 \text { or } 8 \text { or } 9 \text { or } 10 \text { or } 11 \text { or } 12 \\
\text { or } 13 \text { or } 14 \text { or } 15 \text { or } 16 \text { or } 17\end{array}$ \\
\hline \multirow[t]{2}{*}{ Low back pain } & $\begin{array}{l}\text { exp Backache/ } \\
\text { "backache".mp } \\
\text { "back ache".mp } \\
\text { exp Low back pain/ } \\
\text { "low back pain".mp } \\
\text { exp Spinal pain/ } \\
\text { "spinal pain".mp } \\
\text { "back pain”.mp } \\
\text { "lumbago".mp } \\
\text { "lumbar pain".mp } \\
\text { "lower back pain".mp } \\
\text { "vertebral pain".mp } \\
\text { "dorsalgia".mp } \\
19 \text { or } 20 \text { or } 21 \text { or } 22 \text { or } 23 \text { or } 24 \text { or } 25 \text { or } 26 \text { or } 27 \text { or } 28 \\
\text { or } 29 \text { or } 30 \text { or } 31\end{array}$ \\
\hline & \begin{tabular}{|l}
18 and 32 \\
Limit 33 to humans
\end{tabular} \\
\hline
\end{tabular}


Appendix 1 (cont.). Search strategy.

\begin{tabular}{|c|c|}
\hline AMED & Searches \\
\hline Vitamin D & $\begin{array}{l}\text { exp Vitamin D/ } \\
\text { "vitamin D".mp } \\
\text { "vitamin D2".mp } \\
\text { "vitamin D3".mp } \\
\text { "1,25 dihydroxyvitamin D3".mp } \\
\text { " } 25 \text { hydroxycholecalciferol".mp } \\
\text { " } 25 \text { hydroxyvitamin D".mp } \\
\text { "alfacalcidol".mp } \\
\text { "calcidiol".mp } \\
\text { "calcitriol".mp } \\
\text { "calcifediol".mp } \\
\text { "calciferol".mp } \\
\text { "ergocalciferol".mp } \\
\text { "cholecalciferol".mp } \\
\text { exp Cholecalciferols/ } \\
1 \text { or } 2 \text { or } 3 \text { or } 4 \text { or } 5 \text { or } 6 \text { or } 7 \text { or } 8 \text { or } 9 \text { or } 10 \text { or } 11 \text { or } 12 \\
\text { or } 13 \text { or } 14 \text { or } 15\end{array}$ \\
\hline Low back pain & $\begin{array}{l}\text { exp Low Back Pain/ } \\
\text { exp Backache/ } \\
\text { "low back pain".mp } \\
\text { "back pain".mp } \\
\text { "backpain".mp } \\
\text { "backache".mp } \\
\text { "back ache".mp } \\
\text { (lumbar adj5 pain).ti,ab } \\
\text { "lumbar pain".mp } \\
\text { "spinal pain".mp } \\
\text { lumbago.mp } \\
\text { "lower back pain".mp } \\
\text { dorsalgia.mp } \\
\text { "vertebral pain".mp } \\
17 \text { or } 18 \text { or } 19 \text { or } 20 \text { or } 21 \text { or } 22 \text { or } 23 \text { or } 24 \text { or } 25 \text { or } 26 \\
\text { or } 27 \text { or } 28 \text { or } 29 \text { or } 30 \text { or } 31 \text { or } 32\end{array}$ \\
\hline & 16 and 31 \\
\hline Scopus & Searches \\
\hline Vitamin D & 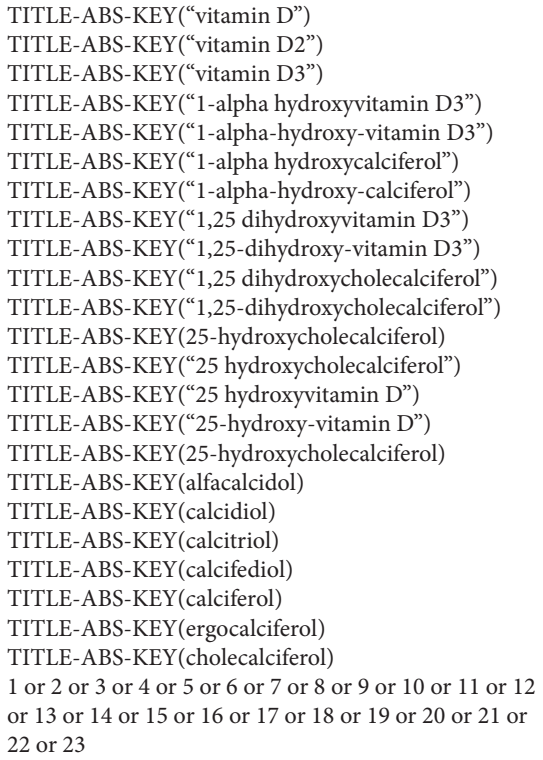 \\
\hline
\end{tabular}

Appendix 1 (cont.). Search strategy.

\begin{tabular}{|c|c|}
\hline \multirow[t]{2}{*}{ Low back pain } & $\begin{array}{l}\text { ALL(“back pain) } \\
\text { TITLE-ABS-KEY(backpain) } \\
\text { ALL(“low back pain") } \\
\text { TITLE-ABS-KEY(backache) } \\
\text { TITLE-ABS-KEY("back ache") } \\
\text { TITLE-ABS-KEY("lumbar pain") } \\
\text { TITLE-ABS-KEY(“spinal pain") } \\
\text { TITLE-ABS-KEY(lumbago) } \\
\text { TITLE-ABS-KEY("lower back pain") } \\
\text { TITLE-ABS-KEY(dorsalgia) } \\
\text { TITLE-ABS-KEY(“vertebral pain") } \\
26 \text { or } 27 \text { or } 28 \text { or } 29 \text { or } 30 \text { or } 31 \text { or } 32 \text { or } 33 \text { or } 34 \text { or } 35\end{array}$ \\
\hline & $\begin{array}{l}24 \text { and } 36 \\
\text { Exclude: "animals" and "animal" }\end{array}$ \\
\hline Web of Science & Searches \\
\hline Vitamin D & $\begin{array}{l}\text { TS=("vitamin D") } \\
\text { TS=("vitamin D2") } \\
\text { TS=("vitamin D3") } \\
\text { TS=(" } 1 \text {-alpha hydroxyvitamin D3") } \\
\text { TS=("1-alpha-hydroxy-vitamin D3") } \\
\text { TS=(" } 1 \text {-alpha hydroxycalciferol") } \\
\text { TS=(" } 1 \text {-alpha-hydroxy-calciferol") } \\
\text { TS=("1,25 dihydroxyvitamin D3") } \\
\text { TS=("1,25-dihydroxy-vitamin D3") } \\
\text { TS=("1,25 dihydroxycholecalciferol") } \\
\text { TS=("1,25-dihydroxycholecalciferol") } \\
\text { TS=(25-hydroxycholecalciferol) } \\
\text { TS=(" } 25 \text { hydroxycholecalciferol") } \\
\text { TS=(" } 25 \text { hydroxyvitamin D") } \\
\text { TS=(" } 25 \text {-hydroxy-vitamin D") } \\
\text { TS=(25-hydroxycholecalciferol) } \\
\text { TS=(alfacalcidol) } \\
\text { TS=(calcidiol) } \\
\text { TS=(calcitriol) } \\
\text { TS=(calcifediol) } \\
\text { TS=(calciferol) } \\
\text { TS=(ergocalciferol) } \\
\text { TS=(cholecalciferol) } \\
1 \text { or } 2 \text { or } 3 \text { or } 4 \text { or } 5 \text { or } 6 \text { or } 7 \text { or } 8 \text { or } 9 \text { or } 10 \text { or } 11 \text { or } 12 \\
\text { or } 13 \text { or } 14 \text { or } 15 \text { or } 16 \text { or } 17 \text { or } 18 \text { or } 19 \text { or } 20 \text { or } 21 \text { or } \\
22 \text { or } 23\end{array}$ \\
\hline \multirow[t]{2}{*}{ Low back pain } & $\begin{array}{l}\text { TS=("back pain") } \\
\text { TS=(backpain) } \\
\text { TS=("low back pain) } \\
\text { TS=(lumbago) } \\
\text { TS=(backache) } \\
\text { TS=("lumbar pain") } \\
\text { TS=("spinal pain") } \\
\text { TS=("lower back pain") } \\
\text { TS=(dorsalgia) } \\
\text { TS=("vertebral pain") } \\
25 \text { or } 26 \text { or } 27 \text { or } 28 \text { or } 29 \text { or } 30 \text { or } 31 \text { or } 32 \text { or } 33 \text { or } 34\end{array}$ \\
\hline & $\begin{array}{l}24 \text { and } 35 \\
\text { TS }=\text { (animals) NOT TS=(humans) } \\
36 \text { NOT } 37\end{array}$ \\
\hline
\end{tabular}


Appendix 2. Modified Downs and Black checklist and individual study scores.

\begin{tabular}{|c|c|c|c|c|c|c|c|c|c|c|c|c|c|c|c|c|}
\hline \multicolumn{13}{|c|}{ Downs and black checklist items (modified for the purpose of our review) } & \multicolumn{4}{|c|}{ Scoring system } \\
\hline \multicolumn{13}{|c|}{ 1. Is the hypothesis/aim/objective of the study clearly described? } & \multicolumn{4}{|c|}{ Yes or no $(1,0)$} \\
\hline \multicolumn{13}{|c|}{ 2. Are the main outcomes to be measured clearly described in the Introduction or Methods section? } & \multicolumn{4}{|c|}{ Yes or no $(1,0)$} \\
\hline \multicolumn{13}{|c|}{ 3. Are the characteristics of the patients included in the study clearly described? } & \multicolumn{4}{|c|}{ Yes or no $(1,0)$} \\
\hline \multicolumn{13}{|c|}{ 4. Are the distributions of principal confounders in each group of subjects to be compared clearly described? } & \multicolumn{4}{|c|}{$\begin{array}{l}\text { Yes (2) } \\
\text { Partially (1) } \\
\text { No (0) }\end{array}$} \\
\hline \multicolumn{13}{|c|}{ 5. Are the main findings of the study clearly described? } & \multicolumn{4}{|c|}{ Yes or no $(1,0)$} \\
\hline \multicolumn{13}{|c|}{ 6. Does the study provide estimates of the random variability in the data for the main outcomes? } & \multicolumn{4}{|c|}{ Yes or no $(1,0)$} \\
\hline \multicolumn{13}{|c|}{$\begin{array}{l}\text { 7. Have actual probability values been reported (e.g. } 0.035 \text { rather than }<0.05 \text { ) for the main outcomes except } \\
\text { where the probability value is less than } 0.001 \text { ? }\end{array}$} & \multicolumn{4}{|c|}{ Yes or no $(1,0)$} \\
\hline \multicolumn{13}{|c|}{$\begin{array}{l}\text { 8. Were the subjects asked to participate in the study representative of the entire population from which } \\
\text { they were recruited? }\end{array}$} & \multicolumn{4}{|c|}{$\begin{array}{l}\text { Yes or no }(1,0) .0 \text { if unable to } \\
\text { determine }\end{array}$} \\
\hline \multicolumn{13}{|c|}{$\begin{array}{l}\text { 9. Were those subjects who were prepared to participate representative of the entire population from which } \\
\text { they were recruited? }\end{array}$} & \multicolumn{4}{|c|}{$\begin{array}{l}\text { Yes or no }(1,0) .0 \text { if unable to } \\
\text { determine }\end{array}$} \\
\hline \multicolumn{13}{|c|}{ 10. If any of the results of the study were based on "data dredging", was this made clear? } & \multicolumn{4}{|c|}{$\begin{array}{l}\text { Yes or no }(1,0) .0 \text { if unable to } \\
\text { determine }\end{array}$} \\
\hline \multicolumn{13}{|c|}{$\begin{array}{l}\text { 11. Were the statistical tests used to assess the main outcomes appropriate? (assume appropriate if unable to } \\
\text { determine) }\end{array}$} & $\begin{array}{l}\text { Yes } \\
\text { dete }\end{array}$ & $\begin{array}{l}\text { no }( \\
\text { ine }\end{array}$ & $0 \mathrm{i}$ & able to \\
\hline 12. Were the main outc & me & ast & & & (va & nd & ble & & & & & & $\begin{array}{l}\text { Yes } \\
\text { dete }\end{array}$ & ne & 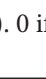 & able to \\
\hline $\begin{array}{l}\text { 13. Were the patients ir } \\
\text { controls (case-control }\end{array}$ & & & & & & & & & & the & (a) & & $\begin{array}{l}\text { Yes } \\
\text { dete }\end{array}$ & $\begin{array}{l}\text { no }( \\
\text { ine }\end{array}$ & 0 & able to \\
\hline $\begin{array}{l}\text { 14. Were study subject } \\
\text { controls (case-control }\end{array}$ & & & & & & & & & & th & ies & & $\begin{array}{l}\text { Yes } \\
\text { dete }\end{array}$ & $\begin{array}{l}\text { no }( \\
\text { hine }\end{array}$ & $0 \mathrm{i}$ & hable to \\
\hline $\begin{array}{l}\text { 15. Was there adequate } \\
\text { drawn? }\end{array}$ & & & & & & & & & & & os w & & $\begin{array}{l}\text { Yes } \\
\text { dete }\end{array}$ & no & $0 \mathrm{i}$ & able to \\
\hline Table 1. 'Downs and & & & & & & & d & vit & $\theta$ & ssib & full & & & & & \\
\hline Author (year) & 1 & 2 & 3 & 4 & 5 & 6 & 7 & 8 & 9 & 10 & 11 & 12 & 13 & 14 & 15 & Total $(/ 16)$ \\
\hline $\begin{array}{l}\text { Abdulmonem A } \\
(2014)\end{array}$ & 1 & 0 & 1 & 0 & 1 & 1 & 1 & 1 & 0 & 0 & 1 & 0 & 1 & 1 & 0 & 9 \\
\hline Alipour M (2015) & 1 & 1 & 1 & 1 & 1 & 1 & 1 & 1 & 0 & 0 & 1 & 0 & 1 & 1 & 0 & 11 \\
\hline Al-Jarallah K (2013) & 1 & 1 & 1 & 0 & 1 & 1 & 0 & 1 & 0 & 0 & 1 & 0 & 0 & 1 & 0 & 8 \\
\hline Baykara B (2014) & 1 & 1 & 1 & 2 & 1 & 1 & 1 & 0 & 0 & 0 & 1 & 1 & 0 & 1 & 0 & 11 \\
\hline Ducher G (2011) & 1 & 1 & 1 & 0 & 1 & 1 & 0 & 1 & 0 & 0 & 1 & 1 & 1 & 1 & 0 & 10 \\
\hline e Silva A (2013) & 1 & 0 & 1 & 2 & 1 & 1 & 1 & 1 & 0 & 0 & 1 & 0 & 1 & 1 & 0 & 11 \\
\hline Ghai B (2015) & 1 & 1 & 1 & 2 & 1 & 1 & 1 & 1 & 0 & 0 & 1 & 1 & 1 & 1 & 0 & 13 \\
\hline Haroon M (2011) & 1 & 1 & 1 & 0 & 1 & 1 & 1 & 1 & 1 & 0 & 1 & 0 & 1 & 1 & 0 & 11 \\
\hline Heidari B (Nov 2014) & 1 & 1 & 1 & 0 & 1 & 1 & 1 & 1 & 0 & 0 & 1 & 1 & 1 & 1 & 0 & 11 \\
\hline Heidari B (2010) & 1 & 1 & 1 & 0 & 1 & 1 & 1 & 1 & 0 & 0 & 1 & 1 & 1 & 1 & 0 & 11 \\
\hline Hicks G (2008) & 1 & 1 & 1 & 2 & 1 & 1 & 1 & 1 & 0 & 0 & 1 & 1 & 1 & 1 & 1 & 14 \\
\hline Johansen J (2013) & 1 & 1 & 1 & 0 & 1 & 1 & 0 & 1 & 0 & 0 & 1 & 1 & 1 & 1 & 0 & 10 \\
\hline Kim T (2013) & 1 & 1 & 1 & 2 & 1 & 1 & 1 & 0 & 0 & 0 & 1 & 1 & 1 & 1 & 1 & 13 \\
\hline Kim T (2012) & 1 & 1 & 1 & 0 & 1 & 1 & 1 & 1 & 0 & 0 & 1 & 1 & 1 & 1 & 0 & 11 \\
\hline Lodh M (2015) & 1 & 1 & 1 & 0 & 1 & 1 & 1 & 1 & 0 & 0 & 1 & 0 & 0 & 1 & 0 & 9 \\
\hline Lotfi A (2007) & 1 & 1 & 1 & 0 & 1 & 1 & 1 & 0 & 0 & 0 & 1 & 1 & 0 & 1 & 0 & 9 \\
\hline Madani M (2014) & 1 & 1 & 1 & 0 & 1 & 1 & 1 & 1 & 0 & 0 & 1 & 1 & 1 & 1 & 0 & 11 \\
\hline
\end{tabular}


Vitamin D and Low Back Pain

Appendix 2 (cont.). Modified Downs and Black checklist and individual study scores.

\begin{tabular}{|l|l|l|l|l|l|l|l|l|l|l|l|l|l|l|l|l||}
\hline Prakash S (2013) & 1 & 1 & 1 & 2 & 1 & 1 & 1 & 0 & 0 & 0 & 1 & 0 & 1 & 1 & 0 & 11 \\
\hline Santos F (2015) & 1 & 1 & 1 & 0 & 1 & 0 & 1 & 1 & 0 & 0 & 1 & 1 & 1 & 1 & 0 & 10 \\
\hline Tanaka S (2013) & 1 & 1 & 1 & 1 & 1 & 1 & 1 & 1 & 0 & 0 & 1 & 0 & 1 & 1 & 1 & 12 \\
\hline Thörneby A (2016) & 1 & 1 & 1 & 2 & 1 & 1 & 1 & 0 & 0 & 0 & 1 & 1 & 1 & 1 & 1 & 13 \\
\hline Waltman N (2009) & 1 & 1 & 1 & 0 & 1 & 0 & 0 & 0 & 0 & 0 & 1 & 1 & 1 & 1 & 0 & 8 \\
\hline Waikakul S (2012) & 1 & 1 & 1 & 0 & 1 & 1 & 0 & 1 & 0 & 0 & 0 & 0 & 1 & 1 & 0 & 8 \\
\hline
\end{tabular}

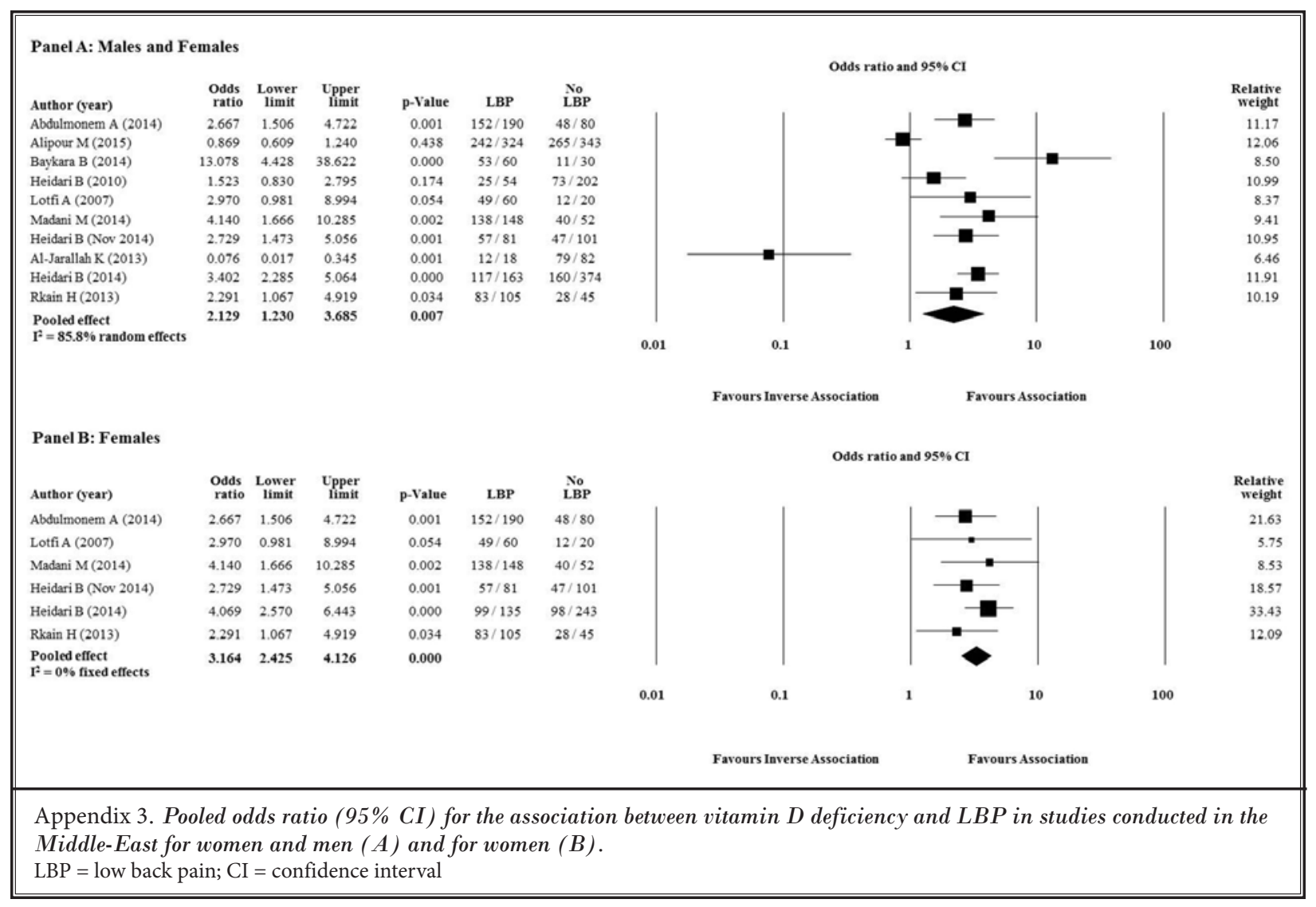


Panel A: Males and Females

$\begin{array}{lrrr}\text { Author (year) } & \begin{array}{r}\text { Odds } \\ \text { ratio }\end{array} & \begin{array}{c}\text { Lower } \\ \text { limit }\end{array} & \begin{array}{r}\text { Cpper } \\ \text { llmit }\end{array} \\ \text { e Silva AV (2013) } & 1.138 & 1.027 & 1.261 \\ \text { Haroon M (2011) } & 1.288 & 0.254 & 6.548 \\ \text { Hicks GE (2008) } & 1.029 & 0.748 & 1.414 \\ \text { Prakash S (2013) } & 5.536 & 1.611 & 19.019 \\ \text { Tanaka S (2013) } & 0.930 & 0.744 & 1.161 \\ \text { Thömeby A (2016) } & 0.316 & 0.063 & 1.575 \\ \text { Ducher G (2011) } & 0.714 & 0.074 & 6.922 \\ \text { Lee KC (2014) } & 1.932 & 1.473 & 2.534 \\ \text { Santos F (2015) } & 0.409 & 0.158 & 1.059 \\ \text { Pooled effect } & \mathbf{1 . 1 4 6} & \mathbf{0 . 8 6 6} & \mathbf{1 . 5 1 6} \\ \mathbf{F}^{2}=\mathbf{7 4 . 6 \%} \text { random effects } & & & \\ & & & \end{array}$

$F^{2}=74.6 \%$ random effects

\begin{tabular}{ccc|}
\multicolumn{4}{c}{ Vit D Deficient / Total } \\
p-Value & LBP & No \\
LBP \\
0.014 & $1580 / 6284$ & $682 / 2992$ \\
0.760 & $6 / 8$ & $156 / 223$ \\
0.862 & $83 / 350$ & $123 / 330$ \\
0.007 & $31 / 35$ & $21 / 36$ \\
0.520 & $216 / 447$ & $513 / 1023$ \\
0.160 & $3 / 41$ & $4 / 20$ \\
0.772 & $2 / 4$ & $7 / 12$ \\
0.000 & $131 / 229$ & $1281 / 3132$ \\
0.066 & $7 / 20$ & $141 / 248$ \\
$\mathbf{0 . 3 3 9}$ & &
\end{tabular}

0.01

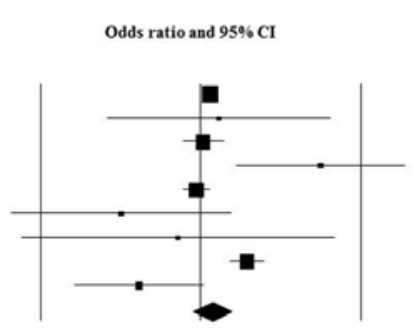

10

1

Panel B: Females

$\begin{array}{lrrr}\text { Author (year) } & \begin{array}{c}\text { Odds } \\ \text { ratio }\end{array} & \begin{array}{c}\text { Lower } \\ \text { limit }\end{array} & \begin{array}{c}\text { Upper } \\ \text { limi }\end{array} \\ \text { e Silva AV (2013) } & 1.138 & 1.027 & 1.261 \\ \text { Hicks GE (2008) } & 0.659 & 0.445 & 0.977 \\ \text { Tanaka S (2013) } & 0.930 & 0.744 & 1.161 \\ \text { Pooled effect } & \mathbf{0 . 9 3 7} & \mathbf{0 . 7 1 9} & \mathbf{1 . 2 2 1} \\ \mathrm{F}^{2}=77.1 \% \text { random effects } & & & \end{array}$

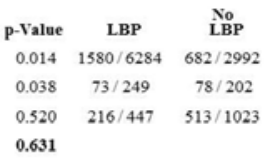

Favours Inverse Association Favours Association

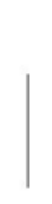

0.1

Favours Inverse Association

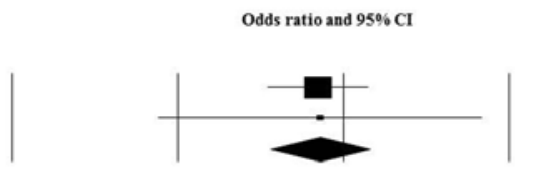

0.1

10
Odds ratio and $95 \% \mathrm{CI}$

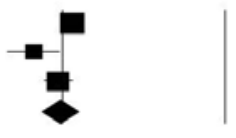

10

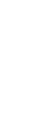

Relative
weight 42.72
22.74 22.74
34.54

Panel C: Males

$\begin{array}{lrrr}\text { Author (year) } & \begin{array}{r}\text { Odds } \\ \text { ratio }\end{array} & \begin{array}{r}\text { Lower } \\ \text { limit }\end{array} & \begin{array}{r}\text { Upper } \\ \text { limit }\end{array} \\ \text { Hicks GE (2008) } & 0.691 & 0.335 & 1.427 \\ \text { Ducher G (2011) } & 0.714 & 0.074 & 6.922 \\ \text { Pooled effect } & \mathbf{0 . 6 9 3} & \mathbf{0 . 3 4 8} & \mathbf{1 . 3 8 3}\end{array}$

$\mathrm{F}=0 \%$ fixed effects
$\mathbf{2}$

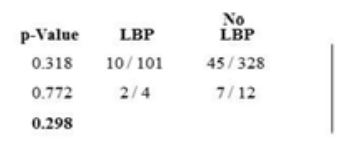

0.01
Relative
weight

9.24

Favours Inverse Association Favours Association

Appendix 4. Pooled odds ratio (95\% CI) for the association between vitamin D deficiency and LBP in studies conducted outside the Middle-East for women and men (A), women (B), and men (C).

$\mathrm{LBP}=$ low back pain; $\mathrm{CI}=$ confidence interval 


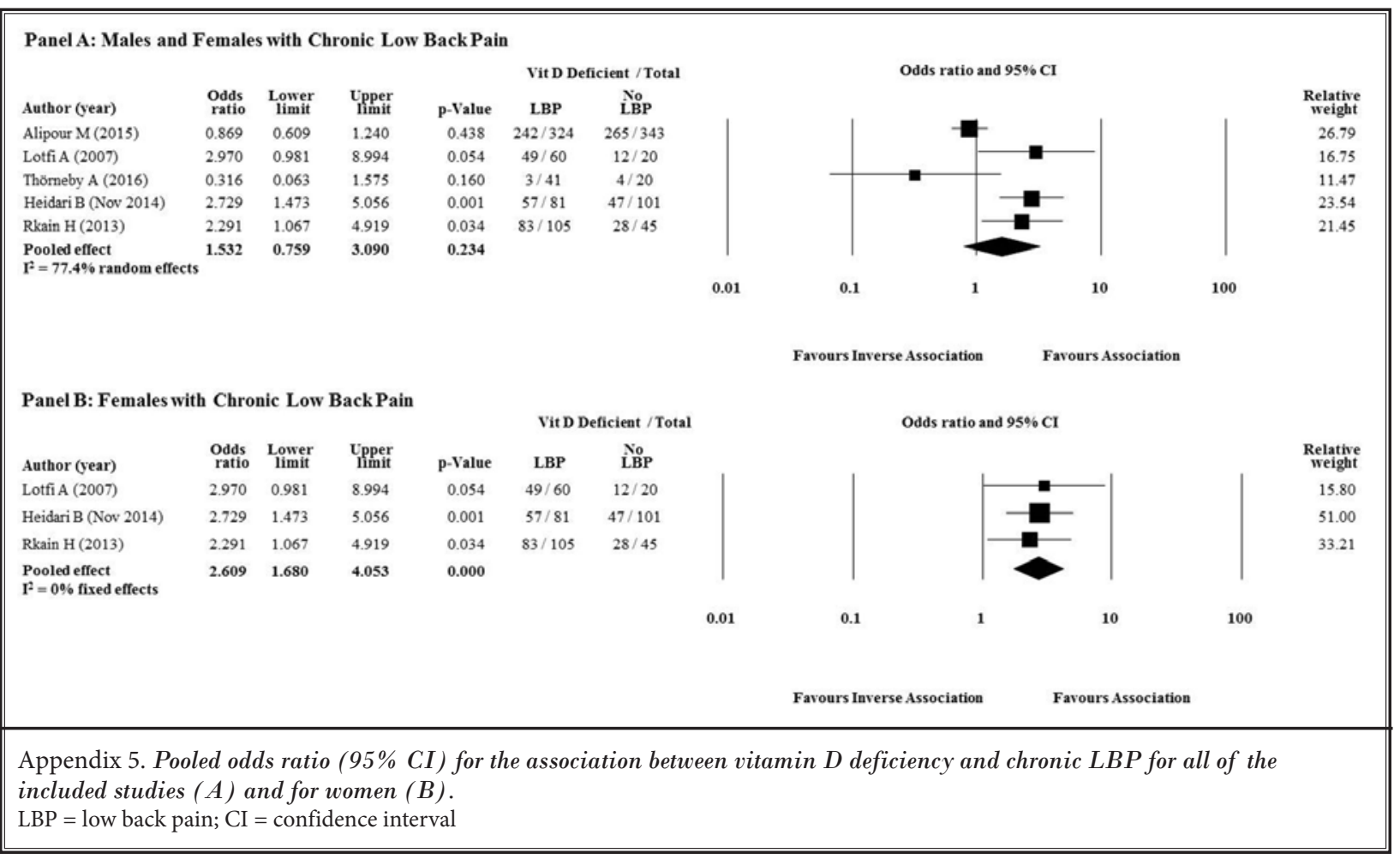

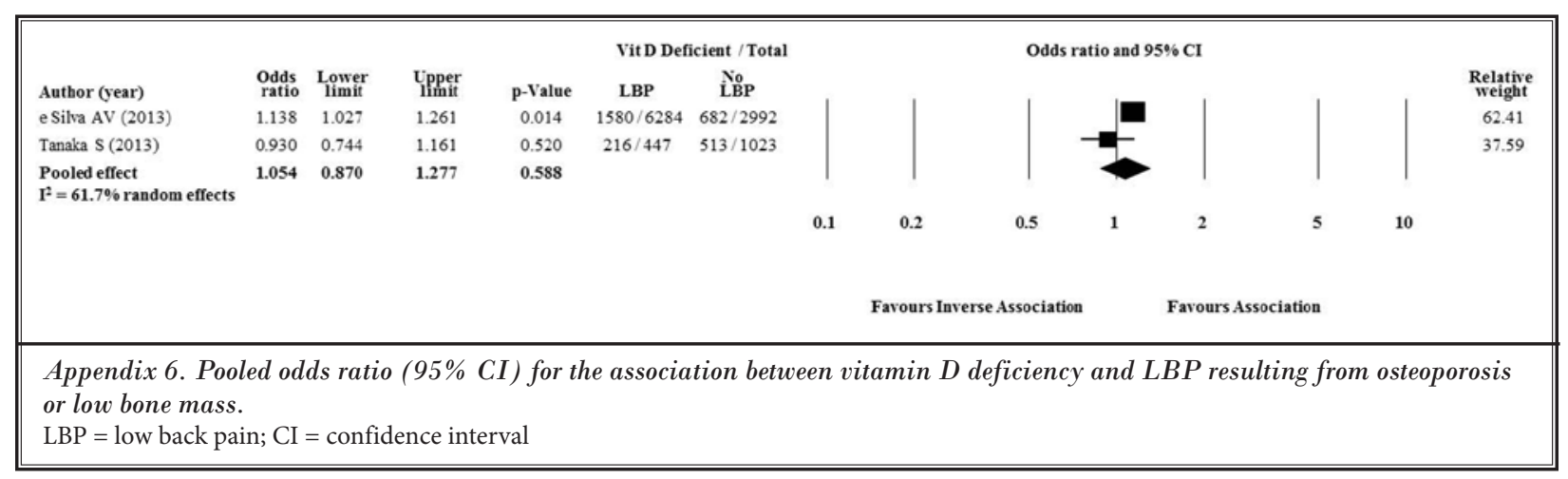


Pain Physician: November/December 2017: 20: 611-640

Appendix 7. Pooled results excluding studies where the full text was not accessible.

\begin{tabular}{|c|c|c|c|c|c|c|c|c|c|c|}
\hline & \multicolumn{5}{|c|}{ ABSTRACTS INCLUDED (Main findings) } & \multicolumn{5}{|c|}{ ABSTRACTS EXCLUDED* } \\
\hline & \multicolumn{10}{|c|}{$\begin{array}{c}\text { Association between Vitamin D deficiency and LBP: excluding Lee KC (2014), Heidari B (2014) and Rkain } \\
\text { H (2013) }\end{array}$} \\
\hline & Pooled OR & $95 \% \mathrm{CI}$ & $\boldsymbol{P}$ & $\mathbf{n}$ & $\mathbf{I}^{2}$ & Pooled OR & $95 \%$ CI & $\boldsymbol{P}$ & $\mathbf{n}$ & $\mathbf{I}^{2}$ \\
\hline All studies & 1.60 & $1.20-2.12$ & 0.001 & 19 & 84.9 & 1.42 & $1.05-1.91$ & 0.023 & 16 & 80.9 \\
\hline Females & 1.83 & $1.26-2.66$ & 0.002 & 9 & 88.4 & 1.45 & $1.04-2.02$ & 0.028 & 7 & 83.0 \\
\hline Males & 1.06 & $0.62-1.81$ & 0.832 & 3 & 44.9 & 0.69 & $0.35-1.38$ & 0.298 & 2 & 0 \\
\hline $\begin{array}{l}\text { Cut-off }<20 \mathrm{ng} / \\
\mathrm{mL}\end{array}$ & 1.25 & $0.89-1.76$ & 0.191 & 13 & 83.3 & 1.05 & $0.77-1.43$ & 0.774 & 11 & 74.3 \\
\hline \multirow[t]{3}{*}{$\begin{array}{l}\text { Cut-off }<10-12 \\
\mathrm{ng} / \mathrm{mL}\end{array}$} & 2.08 & $1.19-3.64$ & 0.010 & 7 & 77.8 & 2.21 & $0.92-5.28$ & 0.075 & 6 & 79.5 \\
\hline & \multicolumn{10}{|c|}{ Association between serum 25(OH)D and LBP: excluding Heidari (2014) and Hussein K (2013) } \\
\hline & Weighted MD & $95 \% \mathrm{CI}$ & $\boldsymbol{P}$ & n & $\mathbf{I}^{2}$ & Weighted MD & $95 \%$ CI & $P$ & $\mathbf{n}$ & $\mathbf{I}^{2}$ \\
\hline All studies & 3.86 & $0.20-7.52$ & 0.039 & 12 & 87.1 & 2.93 & $-1.36-7.21$ & 0.181 & 10 & 84.4 \\
\hline Females & 7.64 & $4.02-11.26$ & $<0.001$ & 4 & 64.6 & 8.06 & $2.07-14.05$ & 0.008 & 3 & 74.9 \\
\hline
\end{tabular}

LBP: low back pain; OR: odds ratio; MD: mean difference; CI: confidence interval; n: number of included studies. ${ }^{*}$ : a number of analyses are missing from this table because the original pooled results didn't include any studies where the full-text was not accessible. 


\section{References}

1. Vos T, Barber RM, Bell B, Bertozzi-Villa A, Biryukov S, Bolliger I, Charlson F, Davis A, Degenhardt L, Dicker D, Duan L, Erskine H, Feigin VL, Ferrari AJ, Fitzmaurice C, Fleming T, Graetz N, Guinovart C, Haagsma J, Hansen GM, Hanson SW, Heuton KR, Higashi $H$, Kassebaum N, Kyu H, Laurie E, Liang X, Lofgren K, Lozano R, Maclntyre MF, Moradi-Lakeh $M$, Naghavi $M$, Nguyen G, Odell S, Ortblad K, Roberts DA, Roth GA, Sandar L, Serina PT, Stanaway JD, Steiner C, Thomas B, Vollset SE, Whiteford H, Wolock TM, Ye P, Zhou M, Ãvila MA, Aasvang GM, Abbafati C, Ozgoren AA, Abd-Allah F, Aziz MIA, Abera SF, Aboyans V, Abraham JP, Abraham B, Abubakar I, Abu-Raddad LJ, Abu-Rmeileh NME, Aburto TC, Achoki T, Ackerman IN, Adelekan A, Ademi Z, Adou AK, Adsuar JC, Arnlov J, Agardh EE, Al Khabouri MJ, Alam SS, Alasfoor D, Albittar MI, Alegretti MA, Aleman AV, Alemu ZA, Alfonso-Cristancho R, Alhabib S, Ali R, Alla F, Allebeck P, Allen PJ, AlMazroa MA, Alsharif $\mathrm{U}$, Alvarez E, Alvis-Guzman N, Ameli O, Amini H, Ammar W, Anderson $\mathrm{BO}$, Anderson HR, Antonio CAT, Anwari $\mathrm{P}$, Apfel $\mathrm{H}$, Arsenijevic VSA, Artaman A, Asghar RJ, Assadi R, Atkins LS, Atkinson C, Badawi A, Bahit MC, Bakfalouni $\mathrm{T}$, Balakrishnan K, Balalla S, Banerjee A, Barker-Collo SL, Barquera S, Barregard L, Barrero LH, Basu S, Basu A, Baxter A, Beardsley J, Bedi N, Beghi E, Bekele T, Bell ML, Benjet C, Bennett DA, Bensenor IM, Benzian $\mathrm{H}$, Bernabe $\mathrm{E}$, Beyene T), Bhala N, Bhalla A, Bhutta Z, Bienhoff $K$, Bikbov B, Abdulhak AB, Blore JD, Blyth FM, Bohensky MA, Basara BB, Borges G, Bornstein NM, Bose D, Boufous $S$, Bourne RR, Boyers $L N$, Brainin $M$, Brauer $M$, Brayne CEG, Brazinova A, Breitborde NJK, Brenner $\mathrm{H}$, Briggs ADM, Brooks PM, Brown J, Brugha TS, Buchbinder R, Buckle GC, Bukhman G, Bulloch AG, Burch M, Burnett R, Cardenas R, Cabral NL, Nonato IRC, Campuzano JC, Carapetis JR, Carpenter DO, Caso V, Castaneda-Orjuela CA, CatalaLopez F, Chadha VK, Chang J-C, Chen $\mathrm{H}$, Chen W, Chiang PP, Chimed-Ochir $\mathrm{O}$, Chowdhury R, Christensen $\mathrm{H}$, Christophi CA, Chugh SS, Cirillo M, Coggeshall M, Cohen A, Colistro V, Colquhoun SM, Contreras AG, Cooper LT, Cooper C, Cooperrider K, Coresh J, Cortinovis M, Criqui MH, Crump JA, Cuevas-Nasu L, Dandona R, Dandona L, Dansereau E, Dantes HG, Dargan PI, Davey G, Davitoiu DV, Dayama A, De la Cruz-Gongora V, de la Vega SF, De Leo D, del Pozo-
Cruz B, Dellavalle RP, Deribe K, Derrett $S$, Des Jarlais DC, Dessalegn M, deVeber GA, Dharmaratne SD, Diaz-Torne C, Ding EL, Dokova K, Dorsey ER, Driscoll $T R$, Duber $H$, Durrani AM, Edmond $K M$, Ellenbogen RG, Endres M, Ermakov SP, Eshrati B, Esteghamati A, Estep K, Fahimi S, Farzadfar F, Fay DFJ, Felson DT, Fereshtehnejad S-M, Fernandes JG, Ferri $\mathrm{CP}$, Flaxman A, Foigt $\mathrm{N}$, Foreman KJ, Fowkes FGR, Franklin RC, Furst T, Futran ND, Gabbe BJ, Gankpe FG, Garcia-Guerra FA, Geleijnse JM, Gessner BD, Gibney KB, Gillum RF, Ginawi IA, Giroud M, Giussani G, Goenka S, Goginashvili K, Gona P, de Cosio TG, Gosselin RA, Gotay CC, Goto A, Gouda HN, Guerrant RI, Gugnani HC, Gunnell D, Gupta R, Gupta R, Gutierrez RA, Hafezi-Nejad $\mathrm{N}$, Hagan $\mathrm{H}$, Halasa $\mathrm{Y}$, Hamadeh RR, Hamavid H, Hammami M, Hankey GJ, Hao Y, Harb HL, Haro JM, Havmoeller R, Hay RJ, Hay S, Hedayati MT, Pi IBH, Heydarpour P, Hijar M, Hoek HW, Hoffman HJ, Hornberger JC, Hosgood HD, Hossain M, Hotez PJ, Hoy DG, Hsairi M, $\mathrm{Hu} \mathrm{H}, \mathrm{Hu} \mathrm{G}$, Huang JJ, Huang C, Huiart $L$, Husseini A, lannarone M, Iburg KM, Innos $\mathrm{K}$, Inoue $\mathrm{M}$, Jacobsen $\mathrm{KH}$, Jassal SK, Jeemon $P$, Jensen $P N$, Jha $V$, Jiang $G$, Jiang $Y$, Jonas JB, Joseph J, Juel K, Kan H, Karch A, Karimkhani C, Karthikeyan G, Katz R, Kaul A, Kawakami N, Kazi DS, Kemp AH, Kengne AP, Khader YS, Khalifa SEAH, Khan EA, Khan G, Khang Y-H, Khonelidze I, Kieling C, Kim D, Kim S, Kimokoti RW, Kinfu Y, Kinge JM, Kissela $B M$, Kivipelto M, Knibbs L, Knudsen AK, Kokubo Y, Kosen S, Kramer A, Kravchenko M, Krishnamurthi RV, Krishnaswami $S$, Defo BK, Bicer BK, Kuipers EJ, Kulkarni VS, Kumar K, Kumar GA, Kwan GF, Lai $\mathrm{T}$, Lalloo R, Lam H, Lan Q, Lansingh VC, Larson $\mathrm{H}$, Larsson A, Lawrynowicz AEB, Leasher JL, Lee J-T, Leigh J, Leung R, Levi M, Li B, Li Y, Li Y, liang J, Lim S, Lin $\mathrm{H}-\mathrm{H}$, Lind M, Lindsay MP, Lipshultz SE, Liu S, Lloyd BK, Ohno SL, Logroscino G, Looker KJ, Lopez AD, Lopez-Olmedo N, Lortet-Tieulent J, Lotufo PA, Low N, Lucas RM, Lunevicius R, Lyons RA, Ma J, Ma S, Mackay MT, Majdan M, Malekzadeh R, Mapoma CC, Marcenes W, March LM, Margono C, Marks GB, Marzan MB, Masci JR, Mason-Jones AJ, Matzopoulos RG, Mayosi BM, Mazorodze TT, McGill NW, McGrath J, McKee M, McLain A, McMahon BJ, Meaney PA, Mehndiratta MM, Mejia-Rodriguez F, Mekonnen W, Melaku YA, Meltzer M, Memish ZA, Mensah G, Meretoja A, Mhimbira FA, Micha R, Miller TR, Mills EJ, Mitchell
PB, Mock CN, Moffitt TE, Ibrahim NM, Mohammad KA, Mokdad AH, Mola GL, Monasta L, Montico M, Montine TJ, Moore AR, Moran AE, Morawska L, Mori R, Moschandreas J, Moturi WN, Moyer M, Mozaffarian D, Mueller UO, Mukaigawara M, Murdoch ME, Murray J, Murthy KS, Naghavi P, Nahas Z, Naheed A, Naidoo KS, Naldi L, Nand D, Nangia V, Narayan KMV, Nash D, Nejjari C, Neupane SP, Newman LM, Newton CR, Ng $M$, Ngalesoni FN, Nhung NT, Nisar MI, Nolte S, Norheim OF, Norman RE, Norrving B, Nyakarahuka L, Oh IH, Ohkubo T, Omer SB, Opio JN, Ortiz A, Pandian JD, Panelo CIA, Papachristou C, Park E-K, Parry CD, Caicedo AJP, Patten SB, Paul VK, Pavlin BI, Pearce N, Pedraza LS, Pellegrini CA, Pereira DM, Perez-Ruiz FP, Perico N, Pervaiz A, Pesudovs K, Peterson CB, Petzold M, Phillips MR, Phillips D, Phillips B, Piel FB, Plass D, Poenaru $D$, Polanczyk GV, Polinder S, Pope CA, Popova S, Poulton RG, Pourmalek F, Prabhakaran D, Prasad NM, Qato D, Quistberg DA, Rafay A, Rahimi K, Rahimi-Movaghar V, Rahman Su, Raju M, Rakovac I, Rana SM, Razavi H, Refaat A, Rehm J, Remuzzi G, Resnikoff S, Ribeiro AL, Riccio PM, Richardson L, Richardus $J \mathrm{H}$, Riederer AM, Robinson M, Roca A, Rodriguez A, Rojas-Rueda D, Ronfani L, Rothenbacher D, Roy N, Ruhago GM, Sabin N, Sacco RL, Ksoreide K, Saha S, Sahathevan R, Sahraian MA, Sampson U, Sanabria JR, Sanchez-Riera L, Santos IS, Satpathy M, Saunders JE, Sawhney M, Saylan MI, Scarborough P, Schoettker B, Schneider IJC, Schwebel DC, Scott JG, Seedat S, Sepanlou SG, Serdar B, Servan-Mori EE, Shackelford K, Shaheen A, Shahraz S, Levy TS, Shangguan S, She J, Sheikhbahaei S, Shepard DS, Shi P, Shibuya K, Shinohara Y, Shiri R, Shishani K, Shiue I, Shrime MG, Sigfusdottir ID, Silberberg DH, Simard EP, Sindi $S$, Singh JA, Singh L, Skirbekk V, Sliwa K, Soljak M, Soneji S, Soshnikov SS, Speyer P, Sposato LA, Sreeramareddy CT, Stoeckl H, Stathopoulou VK, Steckling $\mathrm{N}$, Stein MB, Stein DJ, Steiner TJ, Stewart A, Stork E, Stovner LJ, Stroumpoulis K, Sturua L, Sunguya BF, Swaroop M, Sykes BL, Tabb KM, Takahashi K, Tan F, Tandon N, Tanne D, Tanner M, Tavakkoli M, Taylor HR, Te Ao BJ, Temesgen AM, Have MT, Tenkorang EY, Terkawi AS, Theadom AM, Thomas E, ThorneLyman AL, Thrift AG, Tleyjeh IM, Tonelli M, Topouzis F, Towbin JA, Toyoshima H, Traebert J, Tran BX, Trasande L, Trillini M, Truelsen T, Trujillo U, Tsilimbaris M, 
Tuzcu EM, Ukwaja KN, Undurraga EA, Uzun SB, van Brakel WH, van de Vijver $\mathrm{S}$, Dingenen RV, van Gool $\mathrm{CH}$, Varakin YY, Vasankari TJ, Vavilala MS, Veerman LJ, Velasquez-Melendez G, Venketasubramanian N, Vijayakumar L, Villalpando $S$, Violante FS, Vlassov VV, Waller S, Wallin MT, Wan X, Wang L, Wang J, Wang Y, Warouw TS, Weichenthal S, Weiderpass E, Weintraub RG, Werdecker A, Wessells KRR, Westerman R, Wilkinson JD, Williams HC, Williams TN, Woldeyohannes SM, Wolfe CDA, Wong JQ, Wong $\mathrm{H}$, Woolf AD, Wright JL, Wurtz B, Xu G, Yang G, Yano Y, Yenesew MA, Yentur GK, Yip P, Yonemoto N, Yoon S-J, Younis M, Yu C, Kim KY, Zaki MES, Zhang Y, Zhao Z, Zhao Y, Zhu J, Zonies D, Zunt JR, Salomon JA, Murray CJL; Global Burden of Disease Study 2013 Collaborators. Global, regional, and national incidence, prevalence, and years lived with disability for 301 acute and chronic diseases and injuries in 188 countries, 1990-2013: A systematic analysis for the Global Burden of Disease Study 2013. Lancet 2015; 386:743-800.

2. Salvans MM, Gonzalez-Viejo MA. [Disability by low back pain in Spain from 2000 to 2004]. Med Clin (Barc) 2008; 131:319.

3. Wenig CM, Schmidt CO, Kohlmann T, Schweikert B. Costs of back pain in Germany. Eur J Pain 2009; 13:280-286.

4. Arthritis and Osteoporosis Victoria. A problem worth solving: The rising cost of musculoskeletal conditions in Australia. 2013; http://apo.org.au/node/35957.

5. Keller A, Hayden J, Bombardier C, van Tulder M. Effect sizes of non-surgical treatments of non-specific low-back pain. Eur Spine ] 2007; 16:1776-1788.

6. Taylor JB, Goode AP, George SZ, Cook $\mathrm{CE}$. Incidence and risk factors for firsttime incident low back pain: A systematic review and meta-analysis. Spine J 2014; 14:2299-2319.

7. Hallegraeff JM, Krijnen WP, van der Schans CP, de Greef MH. Expectations about recovery from acute non-specific low back pain predict absence from usual work due to chronic low back pain: a systematic review. J Physiother 2012; 58:165-172.

8. Heneweer H, Staes F, Aufdemkampe G, van Rijn M, Vanhees L. Physical activity and low back pain: A systematic review of recent literature. Eur Spine J 2011; 20:826-845.

9. Croft PR, Papageorgiou AC, Thomas E, Macfarlane GJ, Silman AJ. Short-term physical risk factors for new episodes of low back pain. Prospective evidence from the South Manchester Back Pain Study. Spine (Phila Pa 1976) 1999; 24:1556-1561.

10. Zadro JR, Shirley D, Pinheiro MB, Sánchez-Romera JF, Pérez-Riquelme $F$, Ordoñana JR, Ferreira PH. Does educational attainment increase the risk of low back pain when genetics is considered? A population-based study of Spanish twins. Spine J 2017; 17:518-530.

11. Pinheiro MB, Ferreira ML, Refshauge K, Ordoñana JR, Machado GC, Prado LR, Maher CG, Ferreira PH. Symptoms of depression and risk of new episodes of low back aain: A systematic review and meta-analysis. Arthritis Care Res (Hoboken) 2015; 67:1591-1603.

12. Glover $T L$, Horgas AL, Fillingim RB, Goodin BR. Vitamin D status and pain sensitization in knee osteoarthritis: A critical review of the literature. Pain Manag 2015; 5:447-453.

13. Pereda CA, Nishishinya MB. Is there really a relationship between serum vitamin D (25OHD) levels and the musculoskeletal pain associated with statin intake? A systematic review. Reumatol Clin 2016; 12:331-335.

14. Straube S, Moore RA, Derry S, Hallier E, McQuay HJ. Vitamin D and chronic pain in immigrant and ethnic minority patients-investigation of the relationship and comparison with native Western populations. Int J Endocrinol 2010; 2010:Article ID 753075.

15. Hsiao MY, Hung CY, Chang KV, Han DS, Wang TG. Is serum hypovitaminosis $D$ associated with chronic widespread pain including fibromyalgia? A metaanalysis of observational studies. Pain Physician 2015; 18:E877-E887.

16. e Silva AV, Lacativa PG, Russo LA, de Gregório LH, Pinheiro RA, Marinheiro LP. Association of back pain with hypovitaminosis $D$ in postmenopausal women with low bone mass. BMC Musculoskelet Disord 2013; 14:184.

17. Lotfi A, Abdel-Nasser AM, Hamdy A, Omran AA, El-Rehany MA. Hypovitaminosis $D$ in female patients with chronic low back pain. Clin Rheumatol 2007; 26:1895-1901.

18. Waltman NL, Ott CD, Twiss JJ, Gross GJ, Lindsey AM. Vitamin D insufficiency and musculoskeletal symptoms in breast cancer survivors on aromatase inhibitor therapy. Cancer Nurs 2009; 32:143-150.

19. $\operatorname{Kim} \mathrm{TH}$, Lee BH, Lee HM, Lee $\mathrm{SH}$, Park JO, Kim HS, Kim SW, Moon SH. Preva- lence of vitamin $D$ deficiency in patients with lumbar spinal stenosis and its relationship with pain. Pain Physician 2013; 16:165-176.

20. Heidari B, Shirvani JS, Firouzjahi A, Heidari P, Hajian-Tilaki KO. Association between nonspecific skeletal pain and vitamin D deficiency. Int J Rheum Dis 2010; 13:340-346.

21. Hicks GE, Shardell M, Miller RR, Bandinelli S, Guralnik J, Cherubini A, Lauretani F, Ferrucci L. Associations between vitamin $D$ status and pain in older adults: The Invecchiare in Chianti study. J Am Geriatr Soc 2008; 56:785-791.

22. Calton EK, Keane KN, Newsholme P, Soares MJ. The impact of vitamin D levels on inflammatory tatus: A systematic review of immune cell studies. PLoS One 2015; 10:e0141770.

23. Garcion E, Wion-Barbot N, MonteroMenei CN, Berger F, Wion D. New clues about vitamin $D$ functions in the nervous system. Trends Endocrinol Metab 2002; 13:100-105.

24. Stumpf WE, O'Brien LP. 1,25 $(\mathrm{OH}) 2$ vitamin $D_{3}$ sites of action in the brain. An autoradiographic study. Histochemistry 1987; 87:393-406.

25. Autier P, Boniol M, Pizot C, Mullie P. Vitamin $D$ status and ill health: A systematic review. Lancet Diabetes Endocrinol 2014; 2:76-89.

26. Grossmann RE, Zughaier SM, Liu S, Lyles RH, Tangpricha V. Impact of vitamin D supplementation on markers of inflammation in adults with cystic fibrosis hospitalized for a pulmonary exacerbation. Eur J Clin Nutr 2012; 66:1072-1074.

27. Hopkins MH, Owen J, Ahearn T, Fedirko $V$, Flanders WD, Jones DP, Bostick RM. Effects of supplemental vitamin $D$ and calcium on biomarkers of inflammation in colorectal adenoma patients: A randomized, controlled clinical trial. Cancer Prev Res (Phila) 2011; 4:1645-1654.

28. Sandoughi M, Zakeri Z, Mirhosainee Z, Mohammadi M, Shahbakhsh S. The effect of vitamin $D$ on nonspecific low back pain. Int J Rheum Dis 2015; 18:854-858.

29. Sedighi M, Haghnegahdar A. Role of vitamin $D_{3}$ in treatment of lumbar disc herniation--pain and sensory aspects: Study protocol for a randomized controlled trial. Trials 2014; 15:373.

30. Moher D, Liberati A, Tetzlaff J, Altman DG; PRISMA Group. Preferred Reporting Items for Systematic Reviews and Meta-Analyses: The PRISMA statement. 
Ann Intern Med 2009; 151:264-269, W64.

31. Stroup DF, Berlin JA, Morton SC, Olkin I, Williamson GD, Rennie D, Moher D, Becker BJ, Sipe TA, Thacker SB. Metaanalysis of observational studies in epidemiology: A proposal for reporting. Meta-analysis Of Observational Studies in Epidemiology (MOOSE) group. JAMA 2000; 283:2008-2012.

32. Downs SH, Black N. The feasibility of creating a checklist for the assessment of the methodological quality both of randomised and non-randomised studies of health care interventions. J Epidemiol Community Health 1998; 52:377-384.

33. Higgins JPT, Thompson SG, Deeks JJ, Altman DG. Measuring inconsistency in meta-analyses. BMJ 2003; 327:557-560.

34. Heidari B, Javadian $Y$, Heidari P, Hakimi N, Hajian-Tilaki K, Firouzjahi A. Vitamin $D$ deficiency is associated with nonspecific low back pain in young women, a case-control study. Br J Med Med Res 2014; 4:5053-5061.

35. Kesiktas N, Koser M. Vitamin D levels in young women: A Turkish sample. Osteoporos Int 2011; 22:S721-S722.

36. Santos FC, de Moraes NS, Pastore A, Cendoroglo MS. [Chronic pain in longlived elderly: Prevalence, characteristics, measurements and correlation with serum vitamin D level]. Revista Dor 2015; 16:171-175.

37. Heidari B, Heidari P, Samari E, Jalali MR. [Frequency of vitamin $D$ deficiency in common musculo skeletal conditions]. JBUMS 2014; 16:7-15.

38. Rkain H, Bouaddi I, Ibrahimi A, Lakhdar T, Abouqal R, Allali F, Hajjaj-Hassouni $\mathrm{N}$. Relationship between vitamin $\mathrm{D}$ deficiency and chronic low back pain in postmenopausal women. Curr Rheumatol Rev 2013; 9:63-67.

39. Dionne CE, Dunn KM, Croft PR, Nachemson AL, Buchbinder R, Walker BF, Wyatt M, Cassidy JD, Rossignol M, Leboeuf-Yde C, Hartvigsen J, Leino-Arjas P, Latza U, Reis S, Gil Del Real MT, Kovacs FM, Oberg B, Cedraschi C, Bouter LM, Koes BW, Picavet HS, van Tulder MW, Burton K, Foster NE, Macfarlane G), Thomas E, Underwood M, Waddell G, Shekelle P, Volinn E, Von Korff M. A consensus approach toward the standardization of back pain definitions for use in prevalence studies. Spine (Phila Pa 1976) 2008; 33:95-103.

40. Daly RM, Gagnon C, Lu ZX, Magliano DJ, Dunstan DW, Sikaris KA, Zimmet PZ, Ebeling PR, Shaw JE. Prevalence of vitamin $D$ deficiency and its deter- minants in Australian adults aged 25 years and older: A national, populationbased study. Clin Endocrinol (Oxf) 2012; 77:26-35.

41. Forrest KY, Stuhldreher WL. Prevalence and correlates of vitamin $D$ deficiency in US adults. Nutr Res 2011; 31:48-54.

42. Ducher G, Kukuljan S, Hill B, Garnham AP, Nowson CA, Kimlin MG, Cook J. Vitamin $D$ status and musculoskeletal health in adolescent male ballet dancers a pilot study. J Dance Med Sci 2011; 15:99-107.

43. Haroon M, Bond U, Quillinan N, Phelan MJ, Regan MJ. The prevalence of vitamin $D$ deficiency in consecutive new patients seen over a 6-month period in general rheumatology clinics. Clin Rheumatol 2011; 30:789-94.

44. Lee KC, Khan A, Longworth S, Sell P. Prevalence of vitamin $D$ deficiency in patients presenting with low back pain in an outpatient setting. Eur Spine J 2014; 1:S124-S125.

45. Hampton M, Evans O, Armstrong $S$, Naylor B, Breakwell L, Cole A, Rex Michael AL. Prevalence and significance of vitamin $D$ deficiency in patients with adolescent idiopathic scoliosis requiring corrective surgery. Spine ] 2016; 16:S105.

46. Johansen JV, Manniche C, Kjaer P. Vitamin $D$ levels appear to be normal in Danish patients attending secondary care for low back pain and a weak positive correlation between serum level Vitamin D and Modic changes was demonstrated: A cross-sectional cohort study of consecutive patients with nonspecific low back pain. BMC Musculoskelet Disord 2013; 14:78.

47. Ghai B, Bansal D, Kapil G, Kanukula R, Lavudiya S, Sachdeva N. High prevalence of hypovitaminosis $D$ in Indian chronic low back patients. Pain Physician 2015; 18:E853-E862.

48. Hussein KS, Alkadi H. Vitamin D deficiency is associated with nonspecific skeletal pain in Saudi women. Osteoporos Int 2013; 24:S572.

49. Biering-Sørensen F, Thomsen CE, Hilden J. Risk indicators for low back trouble. Scand J Rehabil Med 1989; 21:151-157.

50. Choi HS, Oh HJ, Choi H, Choi WH, Kim JG, Kim KM, Kim KJ, Rhee Y, Lim SK. Vitamin $D$ insufficiency in Korea--a greater threat to younger generation: The Korea National Health and Nutrition Examination Survey (KNHANES) 2008. J Clin Endocrinol Metab 2011; 96:643-651.

51. Cranney A, Horsley T, O'Donnell S, Wei- ler H, Puil L, Ooi D, Atkinson S, Ward L, Moher D, Hanley D, Fang M, Yazdi F, Garritty C, Sampson M, Barrowman N, Tsertsvadze A, Mamaladze V. Effectiveness and safety of vitamin $D$ in relation to bone health. Evid Rep Technol Assess (Full Rep) 2007; (158):1-235.

52. Guggenbuhl P. Osteoporosis in males and females: Is there really a difference? Joint Bone Spine 2009; 76:595-601.

53. Weaver CM, Gordon CM, Janz KF, Kalkwarf HJ, Lappe JM, Lewis R, O'Karma M, Wallace TC, Zemel BS. The National Osteoporosis Foundation's position statement on peak bone mass development and lifestyle factors: A systematic review and implementation recommendations. Osteoporos Int 2016; 27:1281-1386.

54. Giovannucci E. The epidemiology of vitamin D and cancer incidence and mortality: A review (United States). Cancer Causes Control 2005; 16:83-95.

55. Giovannucci E. Vitamin D and cancer incidence in the Harvard cohorts. Ann Epidemiol 2009; 19:84-88.

56. Munger KL, Zhang SM, O'Reilly E, Hernán MA, Olek MJ, Willett WC, Ascherio A. Vitamin D intake and incidence of multiple sclerosis. Neurology 2004; 62:60-65.

57. Rosendahl-Riise $H$, Spielau U, Ranhoff AH, Gudbrandsen OA, Dierkes J. Vitamin $D$ supplementation and its influence on muscle strength and mobility in community-dwelling older persons: A systematic review and meta-analysis. J Hum Nutr Diet 2017; 30:3-15.

58. Stockton KA, Mengersen K, Paratz JD, Kandiah D, Bennell KL. Effect of vitamin D supplementation on muscle strength: A systematic review and meta-analysis. Osteoporos Int 2011; 22:859-871.

59. Bischoff-Ferrari HA, Dietrich T, Orav EJ, Hu FB, Zhang Y, Karlson EW, DawsonHughes B. Higher 25-hydroxyvitamin D concentrations are associated with better lower-extremity function in both active and inactive persons aged $>$ or $=60$ y. Am J Clin Nutr 2004; 80:752-758.

6o. Wicherts IS, van Schoor NM, Boeke AJ, Visser M, Deeg DJ, Smit J, Knol DL, Lips P. Vitamin D status predicts physical performance and its decline in older persons. J Clin Endocrinol Metab 2007; 92:2058-2065.

61. Boonen S, Lysens R, Verbeke G, Joosten E, Dejaeger E, Pelemans W, Flamaing J, Bouillon R. Relationship between ageassociated endocrine deficiencies and muscle function in elderly women: A cross-sectional study. Age Ageing 1998; 
27:449-454.

62. Annweiler C, Schott-Petelaz AM, Berrut G, Kressig RW, Bridenbaugh S, Herrmann FR, Beauchet O. Vitamin $D$ deficiency-related quadriceps weakness: Results of the Epidemiologie de I'Osteoporose cohort. J Am Geriatr Soc 2009; 57:368-369.

63. Hamberg-van Reenen HH, Ariëns GA, Blatter BM, van Mechelen W, Bongers PM. A systematic review of the relation between physical capacity and future low back and neck/shoulder pain. Pain 2007; 130:93-107.

64. Jørgensen K, Nicolaisen T. Trunk extensor endurance: determination and relation to low-back trouble. Ergonomics 1987; 30:259-267.
65. Holmström E, Moritz U, Andersson M. Trunk muscle strength and back muscle endurance in construction workers with and without low back disorders. Scand J Rehabil Med 1992; 24:3-10.

66. Steiger F, Wirth B, de Bruin ED, Mannion AF. Is a positive clinical outcome after exercise therapy for chronic nonspecific low back pain contingent upon a corresponding improvement in the targeted aspect(s) of performance? A systematic review. Eur Spine J 2012; 21:575-598.

67. Knutsen KV, Madar AA, Brekke $M$, Meyer HE, Natvig B, Mdala I, Lagerløv P. Effect of vitamin D on musculoskeletal pain and headache: A randomized, double-blind, placebo-controlled trial among adult ethnic minorities in Nor- way. Pain 2014; 155:2591-2598.

68. Schreuder F, Bernsen RMD, van der Wouden JC. Vitamin D supplementation for nonspecific musculoskeletal pain in non-Western immigrants: A randomized controlled trial. Ann Fam Med 2012; 10:547-555.

69. Iwamoto J, Takeda T, Ichimura S, Matsu K, Uzawa M. Effects of cyclical etidronate with alfacalcidol on lumbar bone mineral density, bone resorption, and back pain in postmenopausal women with osteoporosis. J Orthop Sci 2003; 8:532-537.

70. Hopewell S, Clarke M, Mallett S. Grey Literature and Systematic Reviews. Publication Bias in Meta-Analysis. John Wiley \& Sons, Ltd, Hoboken 2006, pp 49-72. 